\title{
An image-based model for the sintering of air plasma sprayed thermal barrier coatings
}

\author{
Xun Zhang ${ }^{1,}{ }^{*}$, Alan C.F. Cocks ${ }^{1}$, Yoshifumi Okajima ${ }^{2}$, Kazuma Takeno ${ }^{2}$, Taiji Torigoe ${ }^{2}$
}

${ }^{1}$ Department of Engineering Science, University of Oxford, Parks Road, Oxford OX1 3PJ, UK

${ }^{2}$ Mitsubishi Heavy Industries, Ltd. 1-1 Shinhama, 2-chome, Arai-cho, Takasago, Hyogo, 6768686, Japan

\section{Abstract}

A micromechanical model is developed to predict microstructure evolution and the resultant in-plane modulus increase in a thermal barrier coating (TBC) manufactured by air plasma spray (APS). The model is based on the experimental observation that the main sintering events include the progressive healing of the inter-splat cracks and the coarsening of the columnar grains within the splats. During sintering, the splats undergo Coble creep, which relaxes the constraint on crack healing and therefore sintering. Given its columnar structure, the creep response of the splats is taken to be transversely isotropic. The proposed model is implemented numerically using finite element (FE) meshes that have been developed from cross-sectional micrographs of an as-deposited coating. This allows the evolution of microstructural features directly relevant to the performance of TBCs to be modelled, from which the overall elastic response can also be determined. The curvy nature of the nearly horizontal inter-splat cracks and the intersecting vertical intra-splat cracks explain the low in-plane modulus of the asdeposited TBC and their healing explains the evolution of in-plane modulus during sintering. The model results are validated by comparison with sintering experiments, in terms of the evolution of crack patterns and in-plane modulus. The sensitivity of the model results to the major geometrical parameters is explored and summarised in the form of a sintering map, which identifies the controlling processes as a function of the microstructural and kinetic properties. Finally, the effect of constraint on the overall sintering response is investigated.

Keywords: Sintering; Gas turbine; High temperature; Creep; Cracks

*Corresponding author: Tel. +44 7784679728

E-mail address: xun.zhang@eng.ox.ac.uk 


\section{1. Introduction}

2 Thermal barrier coatings (TBCs) have been widely used in gas turbines to increase the turbine

3 inlet temperature and improve efficiency. State-of-the-art TBC systems generally comprise a

4 ceramic topcoat of $8 \mathrm{wt} . \%$ yttria stabilised zirconia (8YSZ) that provides the thermal insulation,

5 a thermally grown oxide (TGO) and a metallic bond coat deposited on a superalloy substrate

6 [1]. The focus of this study is the behaviour of the topcoat during extended hold periods at

7 elevated temperature.

8 TBC topcoats produced by air plasma spray (APS) have a splat-based microstructure with 9 cracks and pores in between the splats [1]. These microstructural defects over a range of length scales from $1 \mathrm{~nm}$ to $100 \mu \mathrm{m}$ as determined by mercury intrusion porosimetry (MIP) and gassorption techniques $[2,3]$ lead to a decreased thermal conductivity compared to defect free 8YSZ and a lower in-plane Young's modulus (10-50GPa [4-7]), which are both desirable to provide good thermal insulation and strain tolerance and promote a high turbine inlet temperature. However, under service conditions, sintering can occur, which results in healing of the cracks, giving rise to increases in thermal conductivity and in-plane Young's modulus [8]. As a result, a higher thermal stress (and a higher stored strain energy) is induced within the topcoat as the turbine cools down due to the mismatch in the thermal expansion coefficients (CTEs) between the coating and substrate. This stored energy is available to drive delamination of the coating, which occurs when the in-plane Young's modulus of the coating becomes sufficiently high [9]. It is noted that the nano-meter defects sinter fast while the large spherical pores remain largely unchanged during sintering $[2,10]$. Therefore, it is the sintering of the defects on the micron-meter scale that dominates the modulus and therefore the lifetime of the APS TBCs, and that is the focus of this paper. 
(a)

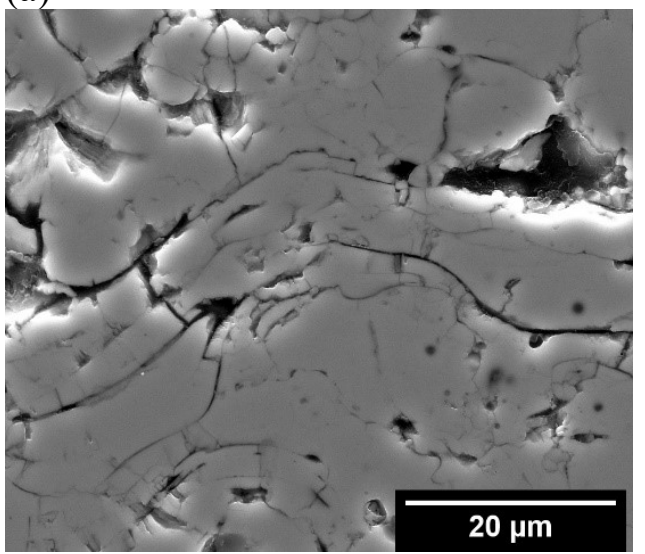

(c)

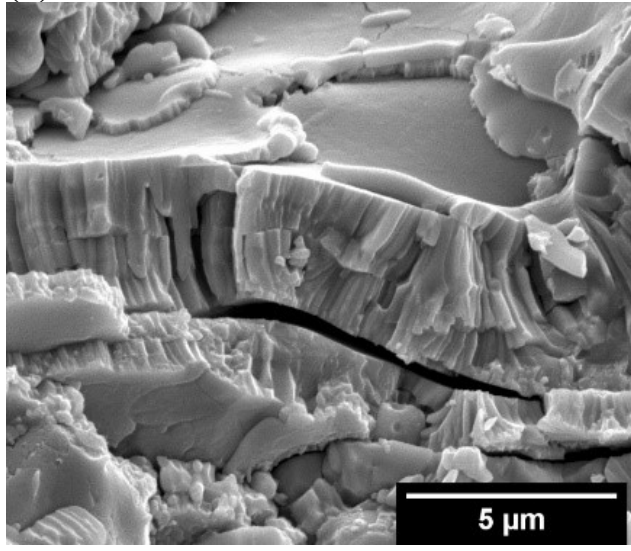

(b)

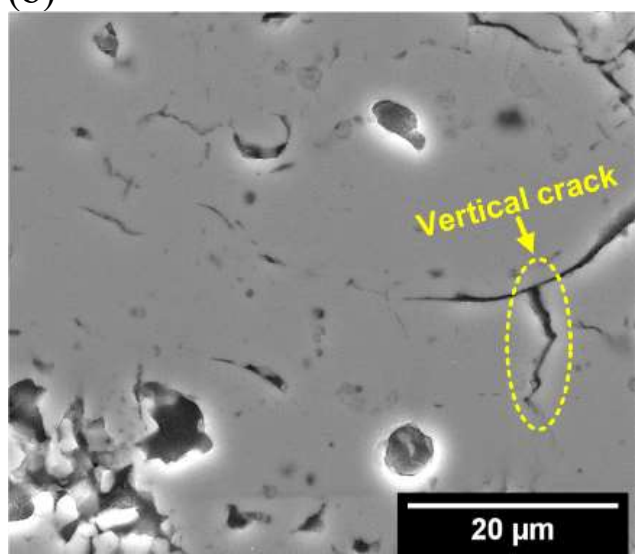

(d)

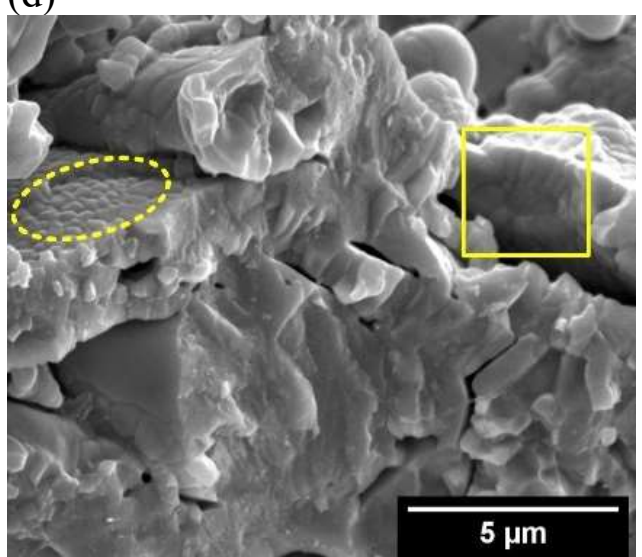

Figure 1 SEM micrographs taken from the polished cross-sections (top row) and fracture surfaces (bottom row) of the APS TBC sample showing (a) curved YSZ splats with mainly horizontal inter-splat cracks in the as-deposited sample; (b) partially healed inter-splat cracks after sintering for $8 \mathrm{hrs}$ at $1400^{\circ} \mathrm{C}$; (c) the curved YSZ splats containing columnar grains with sharp grain boundaries and (d) YSZ splats that become roughened (e.g. the region in the ellipse) and the intra-splat columnar grains that become more spherical after sintering (e.g. the region in the square).

Figure 1 shows an example of the sintering-induced microstructure evolution in an APS TBC.

Cross-sectional micrographs of an as-deposited sample and a section of coating following sintering at $1400^{\circ} \mathrm{C}$ for $8 \mathrm{hrs}$ are shown in Figures 1(a) and 1(b) respectively. The curved splat structure is evident in the as-deposited sample. Almost horizontal undulating cracks follow the shape of the splats. Vertical cracks can be seen, which either extend through or between the splats. Spherical pores measuring $1 \sim 5 \mu \mathrm{m}$ in diameter exist and are intersected by the cracks. After sintering, the horizontal inter-splat cracks have healed partially as shown in Figure 1(b). 
1 Vertical cracks are still evident and can be more open than in the as-deposited coating. The

2 spherical pores have remained largely unchanged after sintering. SEM micrographs taken at surfaces that have been fractured following deposition or sintering, highlight the grain structure evolution. The as-deposited splats shown in Figure 1(c) have a smooth surface and the intrasplat grains are elongated through the splat thickness with sharp grain boundaries. After sintering, these columnar grains have coarsened, and the splat surface has roughened due to thermal grooving at the grain boundaries.

Based on the features of the microstructural evolution described above and exemplified in Figure 1, a number of sintering models have been developed for the splat-based APS TBCs [7, 11-15]. Cipitria et al. [13, 14] have represented the main microstructural features of an APS TBC including the inter-splat pores, intra-splat microcracks and globular voids using a periodic 3D unit cell. Both the free and constrained sintering responses have been modelled. However, their models do not model the evolution of the macroscopic modulus during sintering. In the sintering model developed by Fleck and Cocks [12], it is assumed that the crack surfaces make contact at asperities. How the microstructure evolves is determined by the distribution of cracks and the asperity size and space etc. In each of these models [12-14] the evolution of these major microstructural features is determined using the variational framework described by Cocks et al. [16]. In the isotropic model developed in [12], the gaps between individual splats are represented by a random 3D distribution of planar, circular microcracks, with the faces of each crack bridged by asperities. Inelastic straining due to Coble creep of the splats is considered. This model also predicts the change of the coating's elastic properties as the asperities spread and the cracks close during sintering. In an alternative approach [11], the as-deposited APS TBC is taken as an assembly of discrete splats which are bridged by asperities. The role of the asperity shape and orientation and the degree of the coupling between the coating's in-plane and out-of-plane behaviour have been elucidated. Moreover, the effect of the constraint by the 
1 substrate is also taken into account [11]. These idealised models capture some important

2 features of the sintering response, but the use of simple unit cells does not allow the progressive sintering of cracks that are evident in the micrographs of Figure 1, to be modelled, where cracks sinter initially at points of local contact or adjacent to the tips of the cracks, with these bridged sintered regions gradually spreading along the cracks as new crack faces come into contact as the crack closes. The objective of this paper is to develop a more complete sintering model that is capable of modelling the evolution of microstructure typified by Figure 1, and how this influences the elastic properties of the coating.

In this paper we adopt an image-based approach to model microstructure evolution of a realistic APS TBC and how this influences the elastic properties of the coating. We develop finite element (FE) meshes directly from micrographs similar to those shown in Figure 1. As in the models of Fleck and Cocks $[11,12]$, cracks are assumed to come into contact at asperities. In the model presented here, they progressively sinter as the cracks close, allowing more asperities to come into contact. The resulting computer models provide insight into the sequence of events that determine how the microstructure evolves, including the interaction between different representative microstructural features during sintering, and how this is influenced by the constraint of the substrate. Furthermore, a direct correlation can be established between the evolving microstructure and the increase in in-plane modulus. Moreover, the model can be used for conducting virtual sintering tests, assuming the same initial microstructure, so that the relative importance of different geometric features and internal length scales on the sintering behaviour can be assessed.

\section{The sintering model}

\subsection{Problem statement}

The experimental observations described above and illustrated in Figure 1, suggest that a typical APS TBC sinters mainly via the healing of the inter-splat cracks. Following the models 
1 developed by Cocks and Fleck [7, 11, 12], we assume that these inter-splat cracks are rough

2 and partially in contact at asperities. Driven by changes in interfacial energy, diffusional flow occurs at the asperity level, leading to an increase in the contact area and closure of the bridged crack segments. This brings more asperities on the initially separated crack surfaces into contact. As a result, the inter-splat cracks can heal progressively. In the meantime, the YSZ splats undergo Coble creep at the sintering temperature [17]. The viscosity of the splats depends on the sintering temperature and the size of the intra-splat grains, which increase during sintering. Figure 2 summarises the main processes that are considered in the sintering model developed in this study.

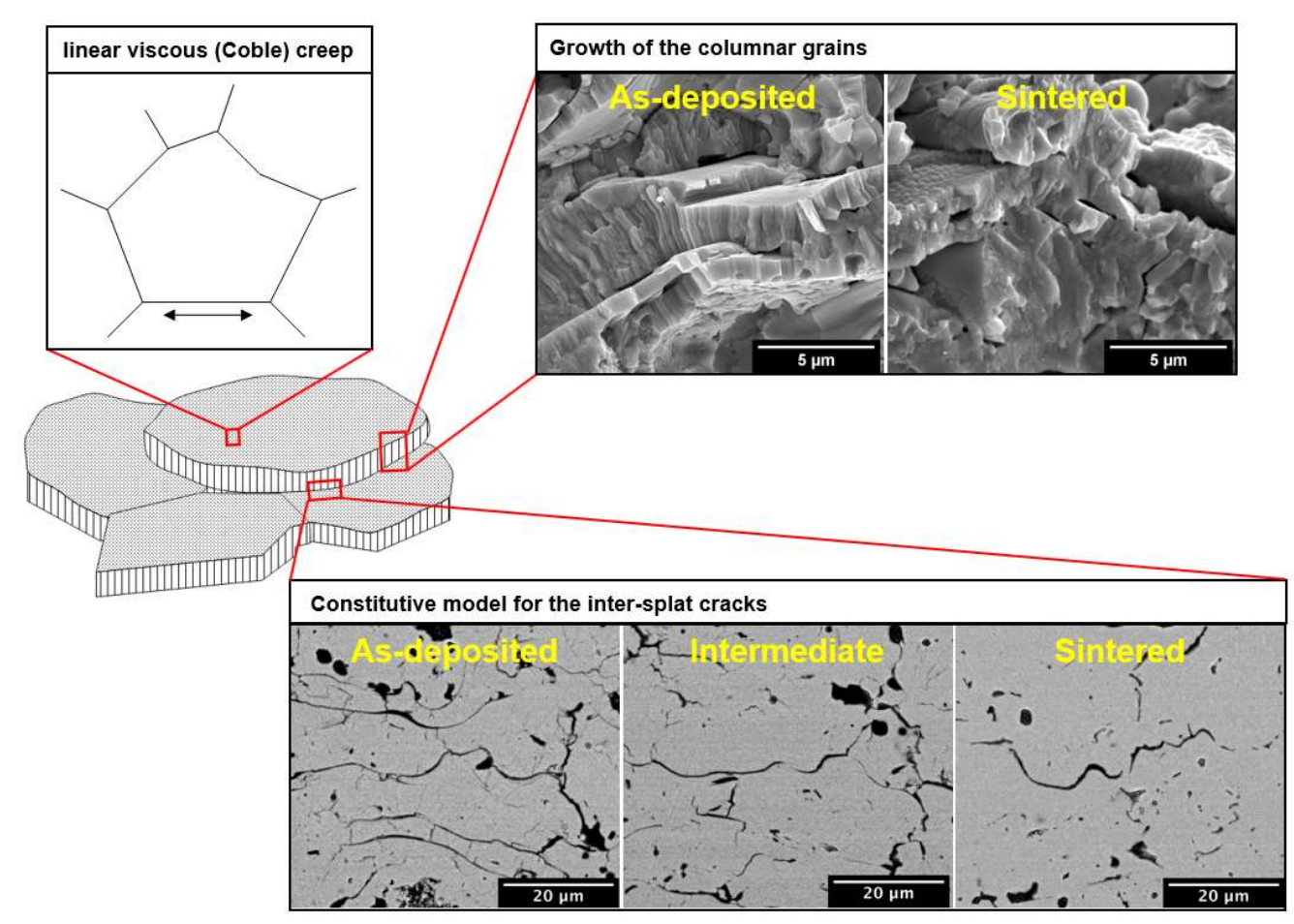

Figure 2 SEM micrographs showing the main microstructural features that evolve during sintering of an APS TBC.

\subsection{Constitutive law for the sintering of inter-splat cracks}

Here we use an image-based approach to simulate the evolution of a realistic microstructure during sintering. Central to this approach is the use of an interface element to explicitly model 
the sintering of the inter-splat cracks. This requires developing a constitutive law for the intersplat cracks as they become bridged by asperities.

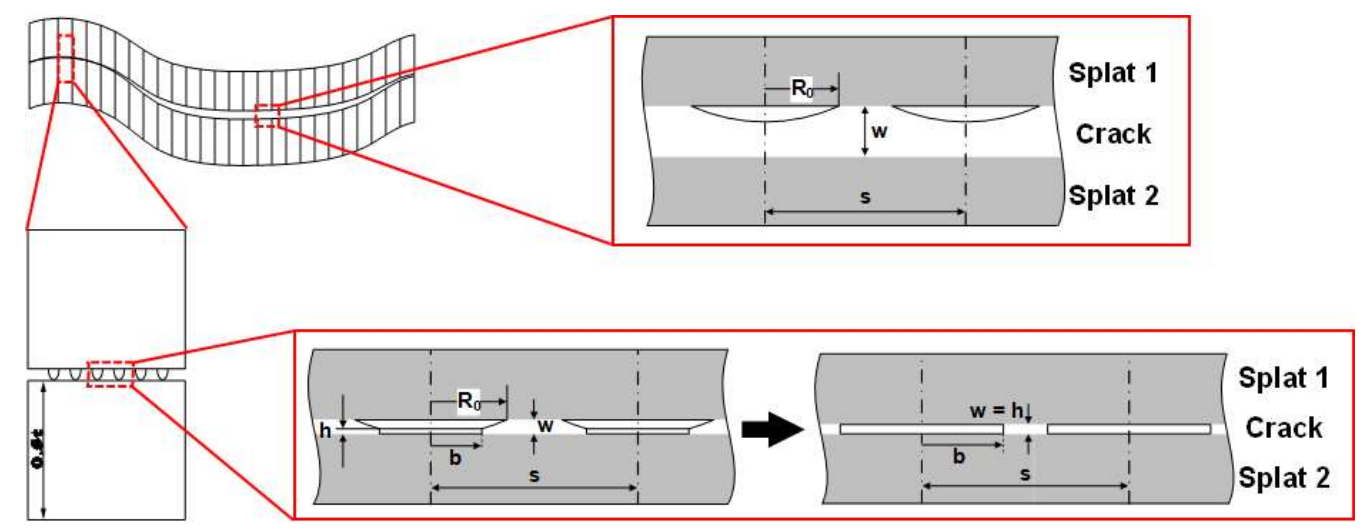

Figure 3 The inter-splat cracks with spherical cap asperities of base radius $R_{0}$ on the crack surfaces. The centre-to-centre distance between the asperities is $s$. The crack opening is $w$. When the opening is larger than the initial asperity height, the crack surfaces are free to displace with respect to each other (upper figure). Cracks are in contact where the opening is smaller than the asperity height e.g. adjacent to a crack tip (lower figure). Sintering leads to an increase in the contact area and a closure of the crack.

Following Fleck and Cocks [12] we assume that the roughness of the inter-splat crack surface has a wavelength $S$ and is modelled by asperities with a centre-to-centre spacing $S$. In practice, a number of geometric features will determine the characteristic wavelength, such as the waviness of the splats and the size of the intra-splat columnar grains, giving rise to multiple wavelengths, each related to a different type of geometric feature. Here, in order to keep the model as simple as possible, we assume that the response can be characterised in terms of a single representative wavelength, which is determined by comparing the predictions with experimental data.

We associate the surface roughness with one surface and the other is kept flat as shown in Figure 3. When the crack surfaces are well separated, they are free to displace with respect to each other (upper diagram in Figure 3). When in contact (e.g. opening smaller than a threshold), 
1 the inter-splat cracks are bridged by the asperities (lower diagram in Figure 3). In their

2 constitutive models for APS TBC sintering, Cocks and Fleck [7, 12] have treated the asperities

3 as circular conical frusta. In practice, the splat surface undulation is milder as illustrated by the

4 micrograph in Figure 1(d). Kumar and Cocks [18] have represented the axisymmetric contacts

5 between the YSZ columns in an EB-PVD TBC using hemispherical asperities. Here, we have

6 modified their approach and treated the asperities as spherical caps so that they have a larger

7 base to height ratio, as the micrograph in Figure 1(d) shows. In the early stage of sintering, the

8 contacting asperity is represented by a truncated spherical cap with a base radius $R_{0}$ connected

9 to a cylindrical region with a base radius $b$ and height $h$ [18]. During sintering, interfacial

10 diffusional flow occurs along the base of the cylinder, leading to an increase in the contact

11 radius $b$ and a decrease in the crack opening $w$, while the base radius of the spherical cap $R_{0}$

12 remains constant $[7,16]$. When $b$ becomes equal to $R_{0}$, the spherical region is eliminated and

the asperity is cylindrical. We assume that it remains cylindrical as it sinters further, decreasing in height and increasing in radius, until $b$ reaches $s / 2$, when full sintering is reached and the crack has completely healed locally. Since volume of an asperity is conserved, the height of the cylinder $h$ and the opening of the asperity-bridged crack $w$ are functions of the contact radius $b$. The detailed expressions are given in Supplementary Data S1.

\subsection{The variational statement} reads:

$$
G_{a}= \begin{cases}S_{\text {truncated-cap }} \gamma_{s}+2 \pi b h \gamma_{s}-\pi R_{0}^{2} \gamma_{s}-\pi b^{2} \gamma_{s}+\pi b^{2} \gamma_{g} & \text { if } b<R_{0} \\ \frac{2 V_{\text {cap }}}{b} \gamma_{s}+\pi b^{2}\left(\gamma_{g}-2 \gamma_{s}\right) & \text { if } b \geq R_{0}\end{cases}
$$




$$
G=\underbrace{G_{a} n_{a}}_{\text {surface energy }}+\underbrace{U n_{a}}_{\text {strain energy }}-\underbrace{T_{n}\left(w-w_{0}\right)}_{\text {potential energy of load }}
$$

2 where $n_{a}=\frac{2 \sqrt{3}}{3 s^{2}}$ is the number of asperities per unit area and $T_{n}$ is the traction normal to the

3 crack surface. The contribution from the strain energy is small compared to the interfacial $4 \quad$ energies and potential energy of the applied traction and therefore can be neglected.

5 For one asperity, diffusional flow along the base and surface gives rise to a rate potential.

6 Assuming that the energy dissipation by diffusion along the asperity surface is much less than

7 that by interfacial diffusion [19], the rate potential for one asperity $\psi_{a}$ can be expressed as:

$$
\psi_{a}=\frac{1}{2 \Phi_{g}} \int_{0}^{b} 2 \pi x j^{2}(x) d x
$$

Mass conservation dictates:

17 where $j(x)$ is the interfacial flux along the interface from the contacts and $\Phi_{g}$ is the effective boundary diffusivity defined as [8]:

$$
\oplus_{g}=\frac{N_{A} D_{0} \delta \Omega}{R T} \exp \left(-\frac{Q_{D}}{R T}\right)
$$

where $N_{A}$ is the Avogadro constant, $D_{0}$ is a reference diffusivity, $\delta$ is the interface thickness, $\Omega$ is the atomic volume, $R$ is the gas constant, $T$ is the absolute temperature and $Q_{D}$ is the activation energy for boundary diffusion.

$$
\dot{w}+\frac{1}{x} \frac{\partial j(x)}{\partial x}=0
$$

Substituting Eqn (5) into Eqn (3) gives:

$$
\psi_{a}=\frac{\pi b^{4} \dot{w}^{2}}{16 \oplus_{g}}
$$


1 The rate equation for the contact radius $b$ is derived using the thermodynamic variational

2 principle as described by Cocks et al. [16]. This is done by determining the value of $\dot{b}$ that

3 minimises the functional $\Phi$ defined as:

$$
\Phi=\dot{G}+\psi
$$

5 where $\dot{G}$ is the rate of change of the Gibbs free energy and $\psi$ is the rate potential per unit 6 volume. The minimisation gives:

$$
\dot{b}=\frac{8 \Phi_{g}\left[T_{n} B(b)-n_{a} A(b)\right]}{n_{a} \pi b^{4} B(b)^{2}}
$$

8 Derivation of Eqn (8) and expressions for $A(b)$ and $B(b)$ are given in Supplementary Data

$9 \mathrm{~S} 1$.

\subsection{Constitutive law for the bridged crack}

11 The normal separation of an asperity-bridged crack segment consists of a sintering part and an

12 elastic part. Therefore, the normal separation rate $\dot{\delta}_{n}$ reads:

$$
\dot{\delta}_{n}=\dot{\delta}_{n}^{e}+\dot{\delta}_{n}^{s}=\left(1-\frac{T_{n}}{k_{n}^{2}} \frac{\partial k_{n}}{\partial \delta_{n}^{s}}\right) \dot{\delta}_{n}^{s}+\frac{\dot{T}_{n}}{k_{n}}
$$

14 where $k_{n}$ is the contact stiffness of the asperities and is defined as a non-linear function of $15 b / s[12]$.

16 Note that $\dot{\delta}_{n}^{s}=\dot{w}$, the rate of change of height of the asperities, which is related to $\dot{b}$ of Eqn (8)

17 through:

$$
\dot{\delta}_{n}^{s}=\dot{w}=B(b) \dot{b}
$$

19 Along the tangential direction, the response is visco-elastic and the shear rate reads: 


$$
\dot{\delta}_{t}=\dot{\delta}_{t}^{e}+\dot{\delta}_{t}^{v}=\frac{\dot{T}_{t}}{k_{t}}+\frac{T_{t}}{k_{t}^{2}} \frac{\partial k_{t}}{\partial \delta_{n}^{s}} \dot{\delta}_{n}^{s}+\frac{T_{t}}{\eta_{t}}
$$

2 where $k_{t}$ and $\eta_{t}$ are the effective shear stiffness and shear viscosity and depend on $b$ and $s$ 3 in a similar way to $k_{n}$.

4

\subsection{Creep response of the splats}

Given their columnar structure, we assume that the YSZ splats undergo transversely isotropic Coble creep at the sintering temperature (e.g. $1000 \sim 1500^{\circ} \mathrm{C}$ [17]). The creep strain rate is linear in the stress:

$$
\dot{\epsilon}_{i j}^{c}=\prod_{i j k l} \sigma_{k l}
$$

where $\Pi_{i j k l}$ is the Coble creep tensor. Its components are determined by the diameter of the columnar grains, the splat thickness and the grain-boundary diffusivity. The components of the creep tensor are given in Supplementary Data S2.

\subsection{Growth of the columnar grains}

The size of the columnar grains needs to be specified in order to determine the creep tensor.

We assume that grain growth follows an ideal growth law:

$$
d^{3}-d_{0}^{3}=k t
$$

The diameters of the intra-splat columnar grains have been measured directly on the SEM images reported in [20]. For each state, 30 measurements were made on randomly picked locations. The grain growth constant $k$ is obtained by fitting the measured grain sizes.

\subsection{Numerical implementation}

The above constitutive law for the bridged crack has been implemented as a user element routine (UEL) in the commercial finite element package ABAQUS 2017. The anisotropic creep law has been implemented as a user material routine (UMAT) for the YSZ splats. An in-house 
1 MATLAB script was developed for generating an FE mesh from a cross-sectional micrograph

2 of an as-deposited APS TBC. It first extracts the crack central lines from the micrographs. The

3

4

5

6 central lines are approximated as segment lines. Following the algorithm developed by Pan et al. [21], the nodes on the segment lines are offset normal to the local tangential direction. Different offset strategies have been adopted for different types of crack. For cracks that extend across the micrograph or connects to pores at both ends (e.g. (1) in Figure 4(b)), a constant base offset equal to the measured mean crack opening is applied. For cracks which are completely contained within the micrograph (e.g. (2) in Figure 4(b)), the base offset is set to zero at the tips and increases linearly to twice the measured mean crack opening towards the middle. For cracks with only one end in the micrograph (e.g. (3) in Figure 4(b)), where they intersect with the micrograph border are taken as the mid-points of the cracks. A random variation with zero mean and the same standard deviation as that of the measured crack opening is added to the offset distance. The nodal information is passed into ABAQUS using the ABAQUS Python scripting interface to generate the finite element (FE) mesh.

Figure 4 (a) to (f) show examples of converting the cross-sectional micrographs for the asdeposited TBC into FE meshes. The mesh shown in Figure 4 (b) contains $~ 9800$ plain strain elements (CPE3 and CPE4) for the YSZ solid and 680 user elements for the cracks. Dummy elements (CPE4) with the same nodal connectivity as the user elements are generated for output and visualisation purposes. 
(a)

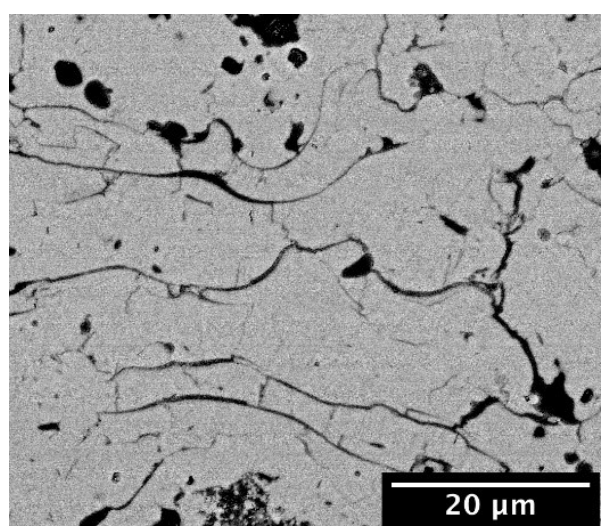

(c)

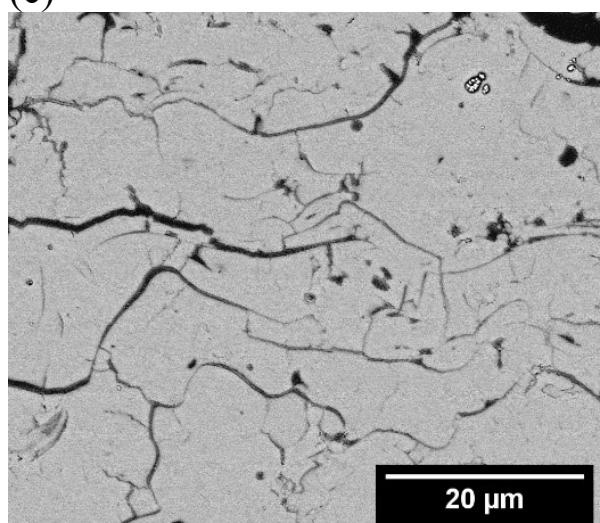

(e)

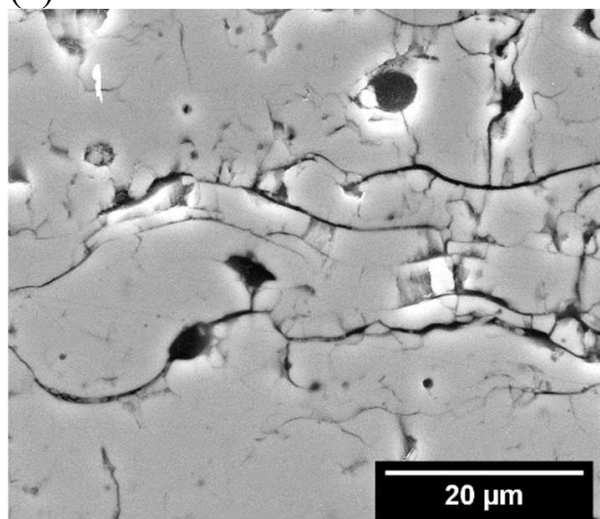

(b)

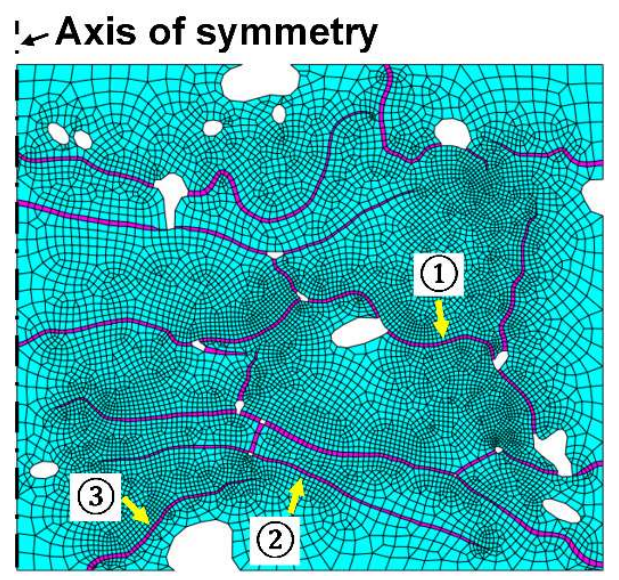

(d)

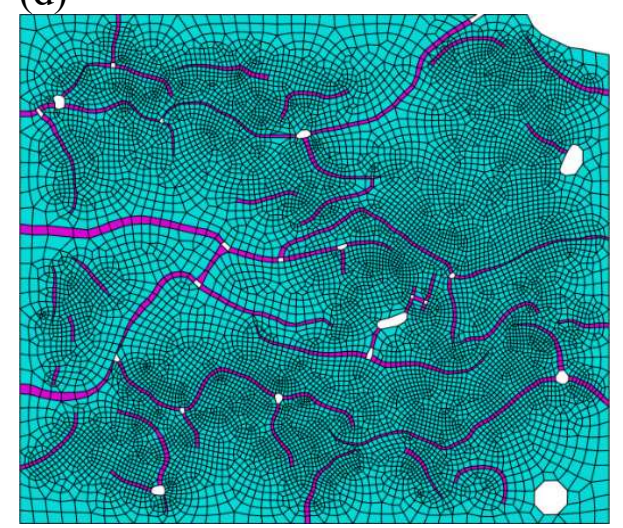

(f)

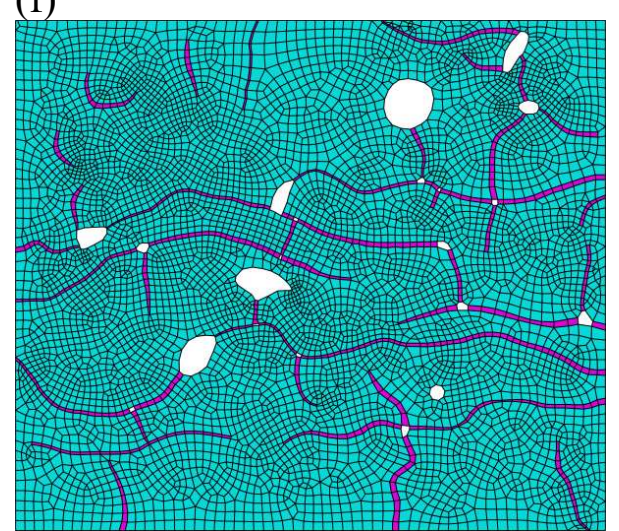

Figure 4 FE mesh generation from the cross-sectional micrographs LHS: SEM micrographs and RHS: the FE meshes developed from the corresponding micrographs.

Unless otherwise stated, the default geometric and material constants for the FE model are

those listed in Table $1[12,13,22-25]$. The model domain is assumed to be symmetric about

the left edge. The effective length of the model domain is therefore twice the micrograph length

and is about $120 \mu \mathrm{m}$, which is large enough to represent a repeating unit of the splats that have

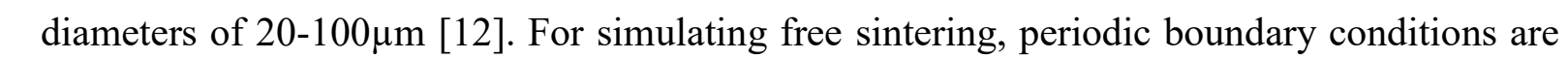


1 applied to the remaining three edges, so that the model domain remains rectangular during

2 sintering. The apparent in-plane modulus is determined by applying a horizontal traction of

$32 \mathrm{MPa}$ to the region and calculating the relative displacement of one vertical face of the unit

$4 \quad$ cell with respect to the opposite face.

Table 1 Default geometrical and material constants used in the FE model.

\begin{tabular}{clc}
\hline \multicolumn{2}{l}{ Geometric parameters } & \\
\hline$R_{0}$ & Base radius of the cap asperity & $1.25 \mu \mathrm{m}$ \\
$s$ & Average asperity spacing & $5.5 \mu \mathrm{m}$ \\
$2 h$ & Average splat thickness & $4 \mu \mathrm{m}$ \\
$d_{0}$ & Initial diameter of the columnar grains & $320 \mathrm{~nm}$ \\
$w_{0}$ & Critical crack opening for asperity bridging & $0.185 \mu \mathrm{m}$ \\
\hline$M^{2}$ aterial constants & \\
\hline$\Phi_{g}$ & Effective boundary diffusivity at $1300^{\circ} \mathrm{C}$ & $1.04 \times 10^{-17} \mathrm{~mm}^{6} \mathrm{~mJ}^{-1} \mathrm{~s}^{-1}$ \\
$Q_{D}$ & Activation energy for boundary diffusion & $370 \mathrm{~kJ} / \mathrm{mol}^{-16} \mathrm{~mm}^{3} / \mathrm{s}$ \\
$k$ & Grain growth constant at $1300^{\circ} \mathrm{C}$ & $2.2 \times 10^{-16}$ \\
$Q_{k}$ & Activation energy for grain growth & $520 \mathrm{~kJ} / \mathrm{mol}^{-1}$ \\
$\gamma_{s}$ & Surface energy (temperature independent) & $1.0 \times 10^{-3} \mathrm{~mJ} / \mathrm{mm}^{2}$ \\
$\gamma_{g}$ & Boundary energy (temperature independent) & $6.4 \times 10^{-4} \mathrm{~mJ} / \mathrm{mm}^{2}$ \\
$E$ & Young's modulus of YSZ bulk & $220 \mathrm{GPa}$ \\
$v$ & Poisson's ratio of YSZ bulk & 0.22 \\
\hline
\end{tabular}

6

7

\section{Model predictions}

\subsection{In-plane modulus of the as-deposited TBC}

Prior to simulating the sintering process, the apparent in-plane Young's modulus for the asdeposited TBC sample is calculated using the image-based FE meshes. In order to account for the variation in microstructure, three micrographs taken from random regions of the asdeposited coating have been meshed. These micrographs together with the FE meshes are shown in Figure 4(a)-(f). An effective in-plane modulus of 20.1 $\pm 5.0 \mathrm{GPa}$ is obtained using the three FE meshes, which is comparable to experimentally obtained values [5, 26-28]. This suggests that the image-based model has captured the principal microstructural features 
1 responsible for the reduced elastic modulus in APS TBCs and validates the simplification of

2 neglecting the finer cracks (nano-meter in size) and large spherical pores. The model can be

4 plane modulus evolution.

5

6

\subsection{Modulus evolution}

We first consider the response when the coating is detached from the substrate and experiences free sinter (i.e. sinters free of any constraint). Figure 5 compares the model predictions for the in-plane elastic modulus from the three representative areas with the experimental measurements reported in [5]. The model predictions capture the general trend in the evolution of the modulus. Note that the detailed evolution depends on the precise starting microstructure. The sensitivity of the exact modulus evolution to the starting microstructure is demonstrated by the different responses obtained from the three meshes. For example, the results from Mesh 2 shows a faster modulus increase at the initial stage of sintering. Comparison of the micrographs in Figure 4 shows that the region represented by Mesh 2 contains more finer cracks. These cracks can sinter fast, giving rise to a faster modulus increase during initial sintering. Averaging in an appropriate way over a number of idealisations generated from different regions of the coating are required to provide an accurate measure of the macroscopic modulus. It should also be noted that while the large (up to $\sim 100 \mu \mathrm{m}$ ) and more spherical pores have been avoided in the micrographs used for running the sintering model, as they remain largely unchanged during sintering [10], they do have an effect on the macroscopic modulus of the coating. A more accurate prediction of the coating's macroscopic modulus and its evolution can be achieved using FE meshes that are developed from micrographs of larger regions where the statistical information on the inter-splat cracks and large pores are representative to the macroscopic case. The scale of the regions modelled here, however, 
1 provide detailed information about how the microstructure evolves during sintering, and how

2 this determines the evolution of modulus.

3

4

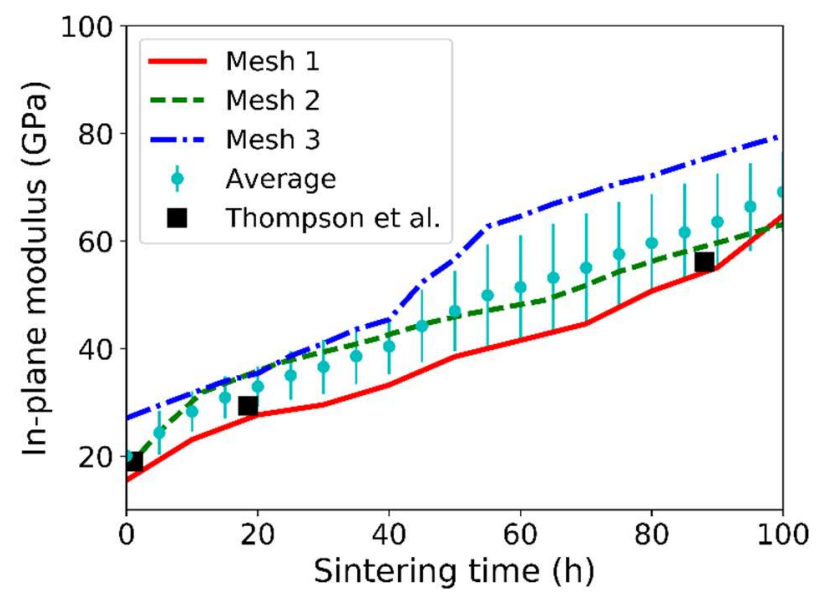

Figure 5 Evolution of the elastic modulus as a function of sintering time at $1300^{\circ} \mathrm{C}$ : experimental data after [5] and the model results for three representative areas. The vertical error bars on the average curve represent the standard deviation of results from the three meshes.

The prediction also depends on the precise choice of internal geometric and material parameters. An example is given in the Supplementary Data S3 where we have calibrated the model prediction against the in-plane modulus measured on the same coatings from which the SEM images shown in Figure 4 have been taken. The measured modulus evolution is successfully reproduced by the models as shown in Figure S-2. We explore the effect of internal geometric and material parameters further below, but here our primary aim is to understand the physical sequence of events that result in sintering and to determine the correlation between microstructural features and modulus evolution during sintering. The modelled evolution of inplane modulus, as shown in Figure 5, is therefore considered as a good starting point. 
(a)

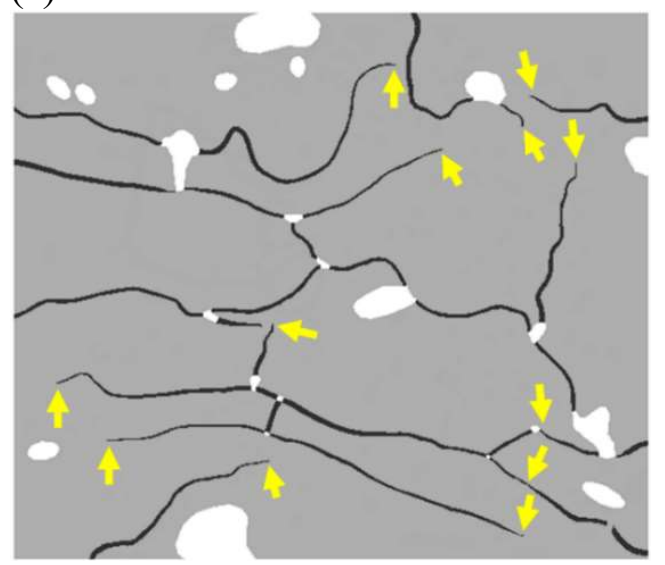

(c)

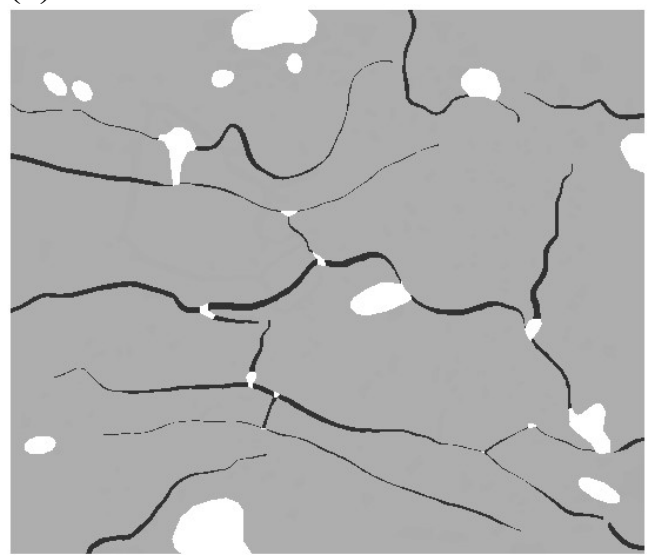

(b)

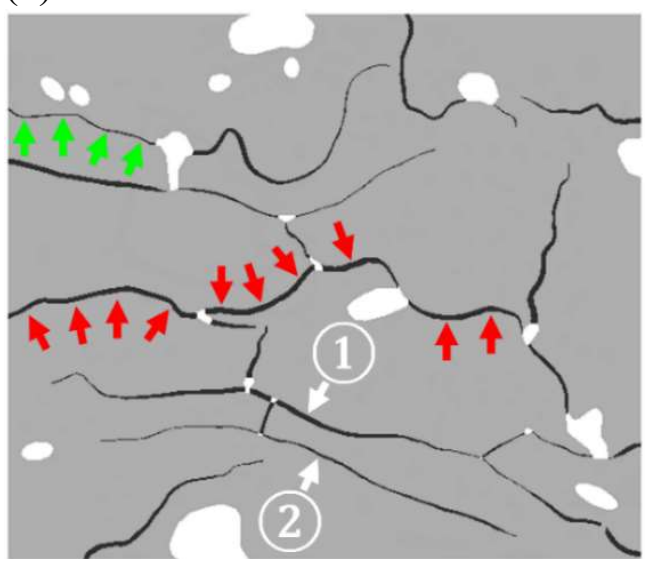

(d)

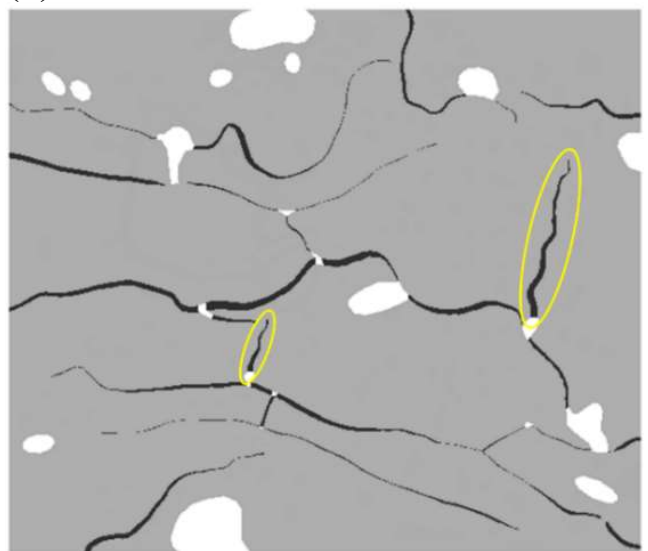

Figure 6 The microstructure of one representative area and how it evolves during sintering: (a) as deposited, where the yellow arrows indicate the crack segments that are initially in contact; (b) after sintering for $50 \mathrm{hrs}$ - the green arrows indicate a crack which is initially open, its faces are brought into contact and they start healing as a result of sintering elsewhere, while the red arrows highlight a crack that has opened more up to this sintering time; (c) after sintering for $75 \mathrm{hrs}$ and (d) after sintering for $100 \mathrm{hrs}$ - the yellow ellipses indicate the almost vertical cracks that have remained open.

It proves instructive in our subsequent discussion to concentrate on the behaviour of one of the microstructures shown in Figure 4. We choose that shown in Figure 4(a). Similar observations can be made concerning the other microstructures analysed. The detailed microstructural evolution processes for the three areas are contained in the Supplementary Videos SV1-SV3.

The simulated evolution of microstructure within the representative area represented by Figure 4 (a) is shown in Figure 6. Unlike other available sintering models for APS TBCs described in 
1 the introduction, where uniform sintering of the inter-splat cracks is assumed, the image-based

2 model predicts the gradual closure of the cracks and provides more details of the sequence of events responsible for sintering. Specifically, sintering starts from the initially bridged crack segments (i.e. opening smaller than the initial asperity height) which is mainly adjacent to the tips of the pre-existing cracks, as indicated by the yellow arrows in Figure 6 (a). Sintering local to these regions pulls the neighbouring crack surfaces close to each other. As a result, new contacts can be gradually established. From Figure 6 (b), asperity contacts have progressed from the tips towards the interior of the cracks. In addition, sintering of the initially contactfree crack (e.g. green arrows in (b)) can be triggered by sintering elsewhere. In this case, a crack can gradually close and asperities are brought into contact as the result of asperities sintering and pulling together the faces of adjacent cracks. Opening of existing cracks is also sometimes observed, as indicated by the red arrows. When two horizontal cracks co-exist close and parallel to each other as is the case of cracks (1) and (2) in Figure 6 (b), sintering occurs first at the narrower crack (i.e. (2)). This is accommodated by opening of the wider crack, (1). As the narrower crack fully sinters, the wider crack begins to sinter, but more slowly due to the larger opening. Also, it is worth noting that the vertical cracks, as highlighted by the yellow ellipses in Figure 6 (d), have remained open throughout the sintering process despite asperities being initially in contact at their tips.

Quantitative image analysis has been performed on cross-sectional micrographs to validate the predicted microstructure evolution. The angular distribution of the total length of the crack segments is plotted in Figure 7 (c) (the details of the image processing are given in Supplementary Data S4), and compared with the model predictions. Note that the experimental micrographs compare representative areas (but not the same areas) before and after sintering, while the results for the simulation results are for the same microstructure. The total length rather than the total number of cracks has been used because the crack number can potentially 
1 increase as a result of incomplete healing (e.g. one crack splits into two as a result of sintering over part of its length).

(a)

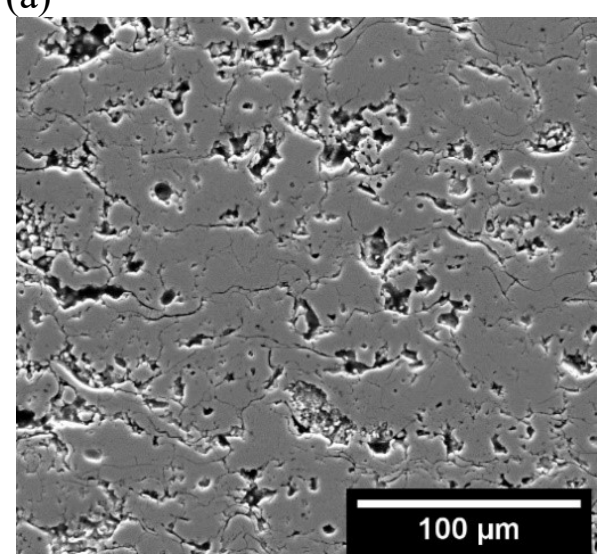

(c)

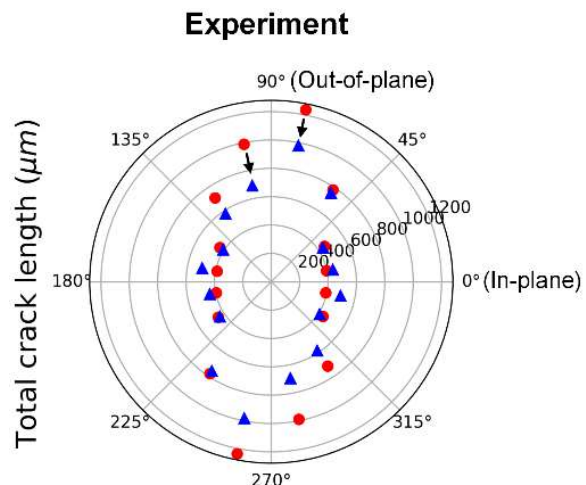

(b)

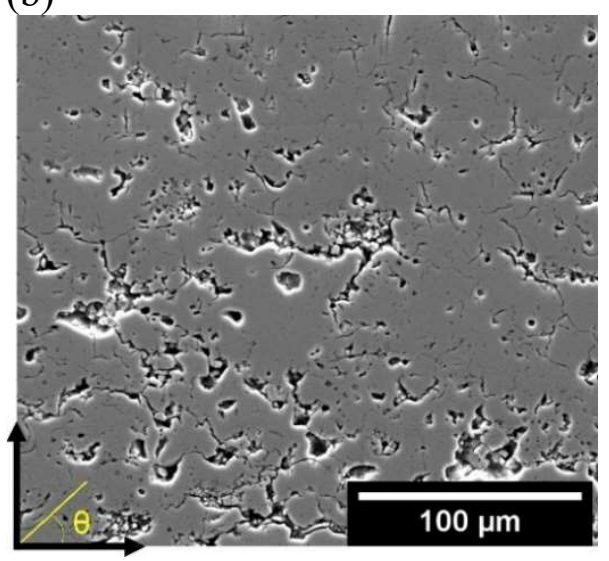

Model

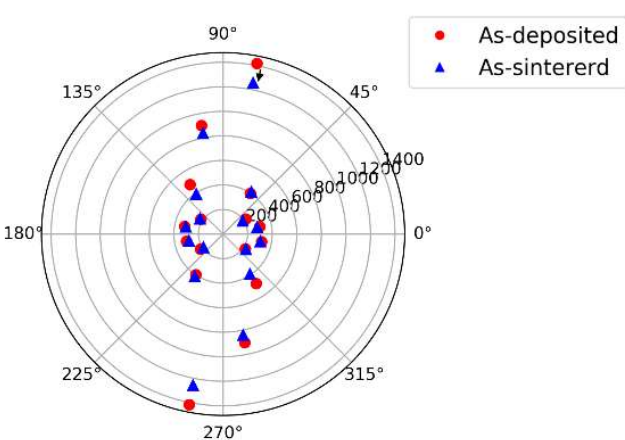

Figure 7 Quantitative analysis of the crack orientation: micrographs showing the region of interest in (a) as-deposited and (b) as-sintered $\left(1400^{\circ} \mathrm{C} 8 \mathrm{hrs}\right)$ samples, from which the crack segments have been analysed - (b) also shows the coordinate system used for the orientation definition; (c) the total crack length as a function of the angle between the coating's in-plane direction and the crack segment normal $(\theta$ as defined in (b)). The black arrows highlight the most significant length reduction.

The experimental results and simulations exhibit similar trends. Figure 7 (c) shows that for the as-deposited TBC, most cracks lie within $\pm 30^{\circ}$ of the coating's in-plane direction. After sintering, the total crack length has reduced in general with the horizontal cracks $\left(\theta \sim 90^{\circ}\right)$ shrinking the most. Interestingly, the total length of the minor vertical cracks $\left(\theta \sim 0^{\circ}\right)$ observed experimentally has increased slightly after sintering, potentially due to the opening of fine vertical cracks that are too fine to resolve in the as-deposited sample, while in the simulations 
1 there is no change in the length of the vertical cracks (note: in the simulations the cracks could 2 open, but not grow).

(a)

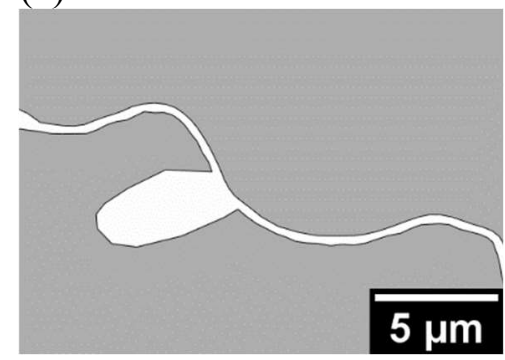

(b)

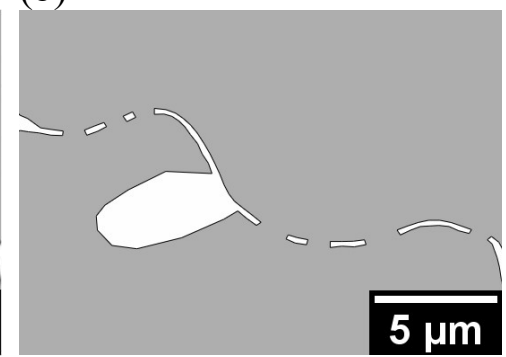

(c)

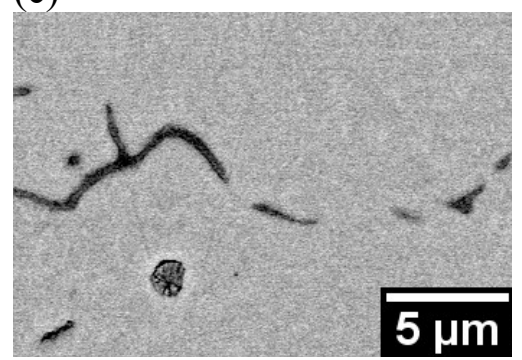

Figure 8 Sintering-induced partial healing of one inter-splat crack: (a) FE model before sintering; (b) after sintering for $8 \mathrm{hrs}$ at $1400^{\circ} \mathrm{C}$, where the user elements that initially represent the crack and have fully sintered merge into the YSZ continuum and (c) SEM cross-sectional micrograph showing one inter-splat crack with a similar morphology to (a), which has become partially healed after sintering.

The progressive healing of one inter-splat crack from the simulation is shown in Figure 8 (a) and (b). Before sintering, this crack is completely open on the image plane. The crack has an irregular profile and its faces are brought into contact at a number of locations as a result of sintering elsewhere. Once in contact, asperity sintering proceeds at these locations and brings the crack faces closer along its entire length. As a result, more regions of the crack come into contact and begin to sinter. This progressive sintering process and prediction of a partially bridged crack is consistent with experimental observations. A typical partially sintered feature illustrating this type of behaviour is shown in Figure 8 (c). It is worth noting that in real APS TBCs, the inter-splat cracks are mostly inter-connected and torturous in 3D [10]. It is possible that what appears to be a completely open crack on the SEM image is bridged somewhere behind the image plane. The partially healed crack in Figure 8 (c) can also be a result of the sintering of the same inter-splat crack at contacts behind the image-plane. Still, this reflects the progressive nature of the inter-splat cracks sintering which is captured by the image-based model. 
2 In the above discussion we have described the general features of the way in which the microstructure evolves during sintering, how this influences the increase in in-plane modulus

4 of the coating and how these are captured by the image-based model. In this sub-section, we

5 use the model to examine the relationship between microstructure and modulus in more detail

6 and correlate changes of modulus with particular features of the microscopic sintering process.

7 In Figure 9 the sintering-induced increase in in-plane modulus is mapped with the

8 microstructure and shows how changes in the stress state and load transfer within the coating

9 are influenced by microstructural changes.

10 Figure 9 (b) shows the in-plane stress (S11) distribution within the as-deposited sample when

11 a horizontal traction of $2 \mathrm{MPa}$ is applied to the right-hand face. The alternating tensile and compressive stresses in Figure 9 (b) suggests that the nearly horizontal wavy cracks put the YSZ splats under bending. This alone can degrade the apparent in-plane modulus significantly. Moreover, the vertical cracks can unload the splats locally (e.g. the region inside the red dash line). The extent of the unloading depends strongly on their relative position with respect to the horizontal cracks. For example, a through-splat vertical crack that connects two horizontal inter-splat cracks as indicated by the white arrow in the middle of Figure 9 (b), though short, tends to unload the splat along its whole length. This extensive unloading of a splat, coupled with the resulting enhancement of stress in adjacent splats provides an interconnected path across the unit cell, which results in a significant reduction of the in-plane modulus compared to that of the bulk material. 
(a)

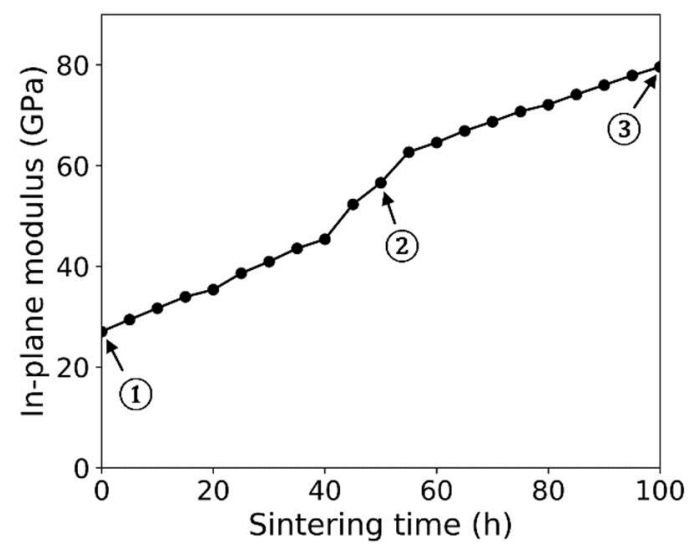

(c)

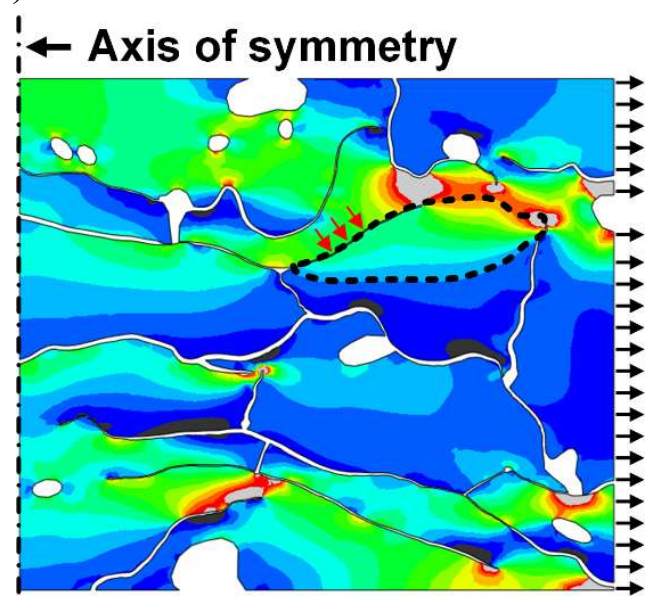

(b)

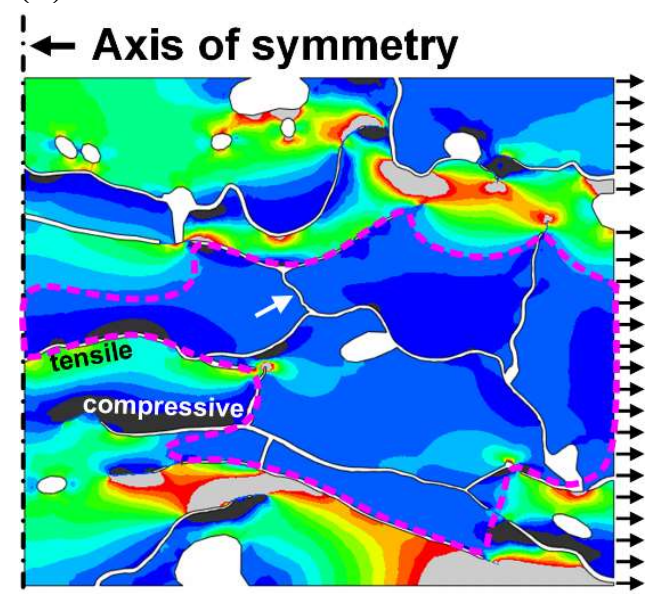

(d)

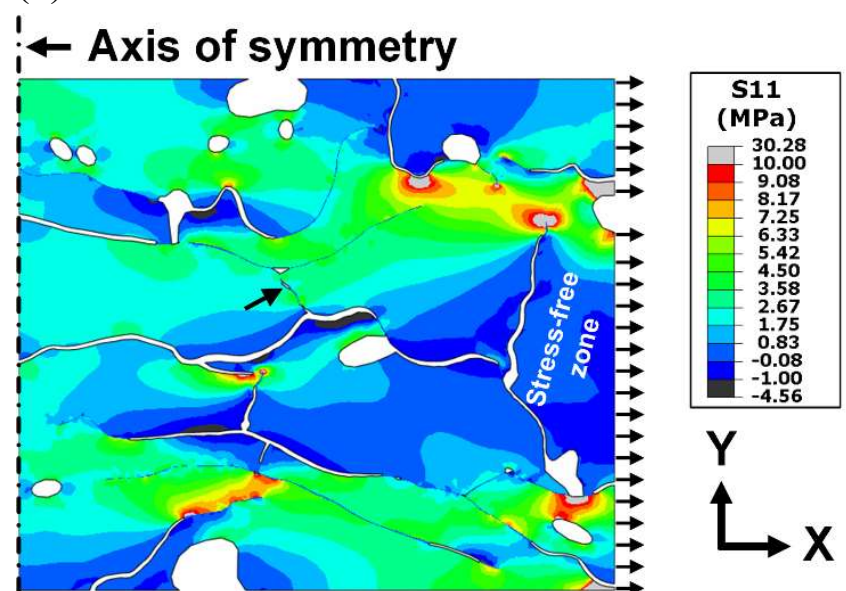

Figure 9 Model prediction of the evolution of the apparent in-plane modulus as a result of sintering at $1300^{\circ} \mathrm{C}$ and the corresponding microstructure at different times, indicated by the arrows in (a) - in-plane stress (S11) contours are superimposed on the microstructure plots (b) to (d) when a surface traction of 2 $\mathrm{MPa}$ is applied to the right edge in the $\mathrm{x}$-direction.

With increasing time at temperature, local regions of the nearly horizontal wavy crack faces come into contact, leading to localised sintering, e.g. as indicated by the red arrows in Figure 9(c). Load can be transferred into a splat at these local pinning points (e.g. the region outlined by the black dashed line begins to carry load), promoting a reduction of the bending stresses and a reduction in the length over which a splat is unloaded by a vertical crack. Localised sintering at a small number of contact points can significantly enhance the in-plane modulus. As noted earlier, vertical cracks tend to remain open longer and sinter much slower than the near horizontal cracks. During the initial stages of sintering the increase in modulus is due to 
1 localised sintering of the wavy horizontal cracks, which might be counter intuitive. With

2 increasing time at temperature, more extensive healing of the nearly horizontal cracks occurs and some of the vertical cracks can close and heal (e.g. the small vertical crack indicated by the black arrow in Figure 9(d)), resulting in a more uniform distribution of stress within the coating, with only the regions immediately next to the remaining vertical cracks still stress free, as shown in Figure 9(d).

\section{Effect of material length scales and constraint on sintering}

\subsection{Sensitivity of the model to internal length-scales}

The rate at which, and the way that, the microstructure evolves is sensitive to the length scales of the different geometric features in the model and the timescales for the kinetic processes, particularly the relative time scales for asperity sintering and Coble creep [11, 13]. For example, if the viscosity of the matrix is small, the sintering of a crack can be readily accommodated by Coble creep of the surrounding matrix and there is limited influence on the sintering of adjacent cracks, but at high viscosities there is limited creep and closing of a crack due to sintering of contacting asperities is often accommodated by the opening of adjacent cracks. In this section, the effect of the relevant time and length-scales on the model results is investigated. As before, we illustrate the influence of these different length and time scales by evaluating how they influence the evolution of the microstructure shown in Figure 4 (a).

\subsubsection{Effect of asperity spacing}

The effect of asperity spacing on the overall sintering response (quantified in terms of the inplane modulus) is explored in Figure 10. Given the same initial microstructure, a smaller asperity spacing leads to a higher initial in-plane modulus. This is because a smaller asperity spacing gives the initially bridged crack segments a higher contact stiffness [12]. 
(a)

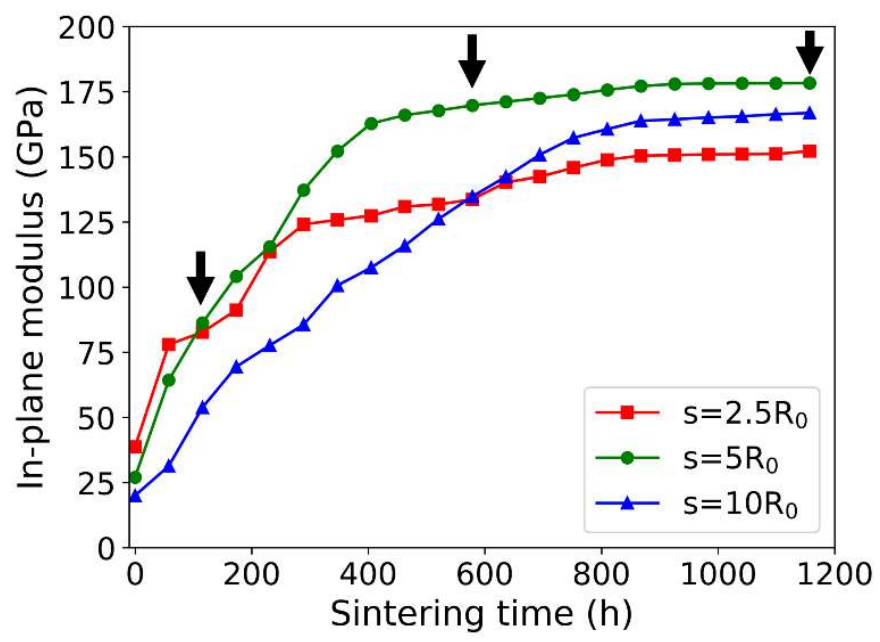

(b)
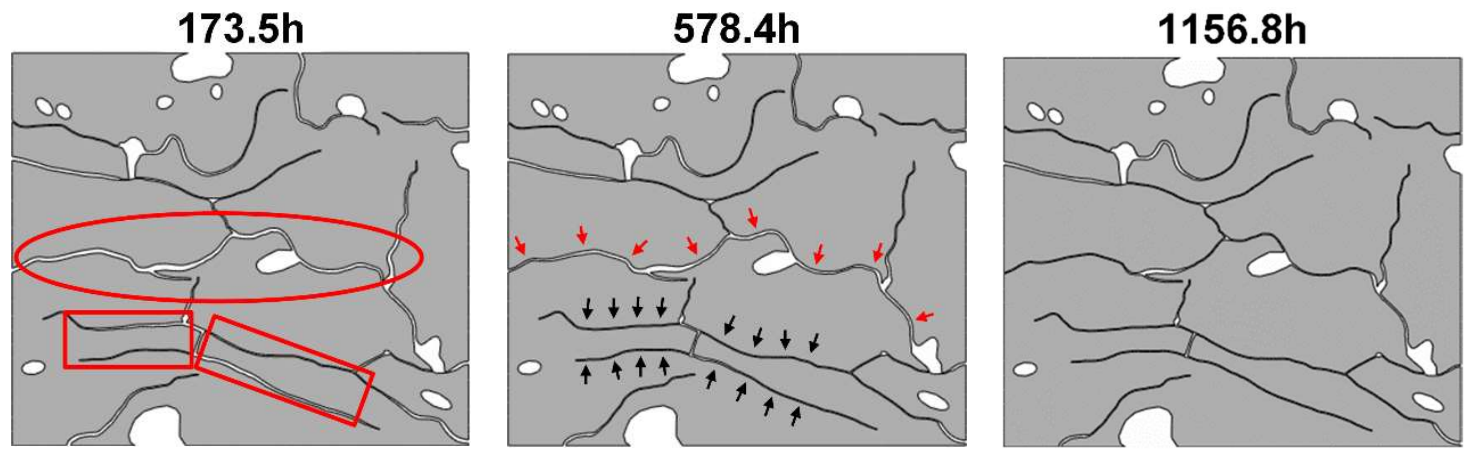

(c)
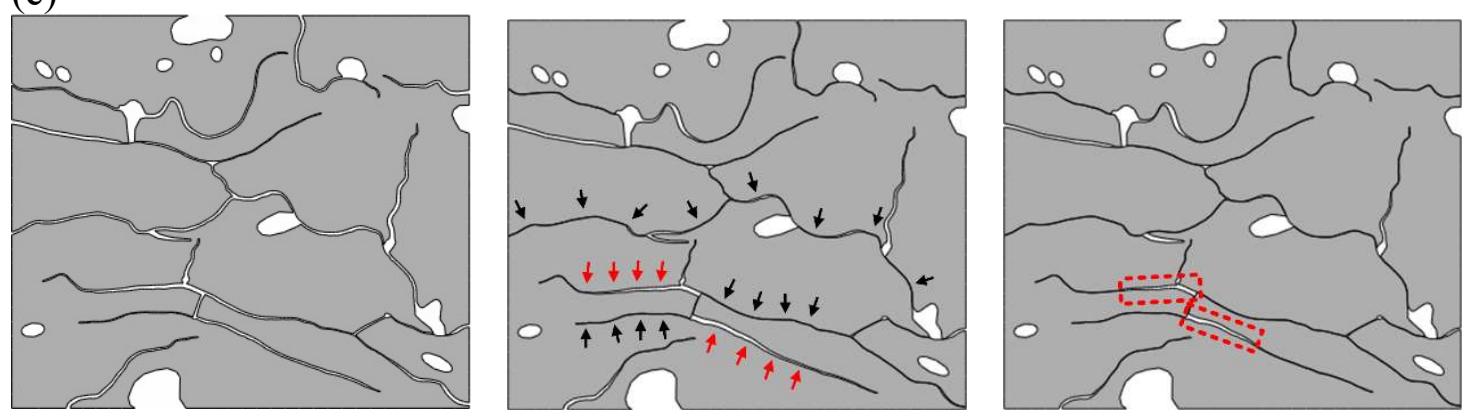

Figure 10 Evolution of the apparent in-plane modulus for different asperity spacings

(a) and the corresponding microstructure predicted at different stages of sintering as indicated by the arrows for (b) $s=2.5 R_{0}$ and (c) $s=10 R_{0}$. The black arrows indicate the crack segments that have healed while the red arrows indicate the crack segments that either open or do not sinter.

The sintering response doesn't change monotonically with the asperity spacing as predicted by models based on a simplified microstructure [7]. For the largest value of $s$ considered in Figure 10 , the in-plane modulus gradually increases towards a 'plateau' about $75 \%$ of the bulk value.

Smaller asperity spacings give a more rapid sintering responses initially. However, for the case 
1 of $s=2.5 R_{0}$, the rate of sintering slows significantly after a short initial rapid period. This is

2 followed by a second rapid sintering stage and finally a gradual increase to a plateau slightly

3 lower than the other two cases.

$4 \quad$ Figure 10 (b) and (c) show that the microstructure has evolved differently for the two asperity 5 spacings. For the smaller values of $s$, the nearly parallel cracks to the bottom of the analysed 6 region (indicated by rectangles in Figure $10(\mathrm{~b})$ ) have healed almost simultaneously while for

7 larger $s$, one crack heals at the expense of the other, which opens (see the different response 8 as indicated by the red and black arrows in Figure $10(\mathrm{~d}))$. The opened cracks only begin to 9 heal in the later stage of sintering and haven't healed fully at the end of the simulation. The residual crack segments are marked by the red dashed rectangle in Figure 10 (d).

Increasing the asperity spacing essentially decreases the overall sintering force and therefore reduces the relaxation rate by Coble creep. As an alternative relaxation mechanism, opening of nearby cracks is favoured when the asperity spacing is sufficiently large. It is also noticed that a larger asperity spacing facilitates the healing of initially un-bridged cracks (e.g. that identified by an ellipse in Figure 10 (b)). Indeed, this crack has almost completely healed after $578.4 \mathrm{~h}$ for $s=10 R_{0}$ while it has remained un-bridged for $s=2.5 R_{0}$. Recall that the healing of initially unbridged cracks can only be triggered when a section of the crack closes sufficiently as a result of asperity sintering elsewhere for asperities on opposite crack faces to touch. For the larger asperity spacing, full sintering of the asperities initially in contact can give rise to a larger closure displacement. As a result, contacts can be established earlier for the initially un-bridged cracks as is seen for the crack highlighted by the ellipse. The late establishment of contacts for initially un-bridged cracks may also explain the decreased sintering rate (modulus increase rate) during the early stages of sintering for $s=2.5 R_{0}$. 
1 For real APS TBCs, the asperity spacing relates to the waviness of the splats and the surface

2 roughness of the splats, which evolves with sintering due to thermal grooving at the grain boundaries. As a result, there are multiple length scales associated with the size and spacing of the asperities. These microstructural features could potentially be measured experimentally, and the model extended to account for a distribution of asperity sizes and spacing. Here we have based the model on a single size and spacing for the asperities, which are treated as parameters that are determined by calibrating the predictions of the model against experimental data. This section provides an indication of the sensitivity of the predicted microstructure evolution and the resultant in-plane modulus on the asperity spacing.

\subsubsection{Effect of splat thickness}

The viscosity of a YSZ splat is directly related to the splat thickness trough the Coble creep tensor of Eqn (12). Figure 11 (a) shows the effect of changing the splat thickness on the inplane modulus evolution. A thinner splat leads to a faster sintering on average. For $h=2 \mu m$, the in-plane modulus increases rapidly until a plateau is reached. For $h=10 \mu m$, a two-stage response is observed with a rapid increase (still slower than that for $h=2 \mu \mathrm{m}$ ) for the first $\sim 100 \mathrm{~h}$ followed by a more gradual increase. This response is consistent with the experimental observations reported by Thompson et al. from their sintering experiments at $1400^{\circ} \mathrm{C}$ [5]. When creep is deactivated (i.e. $h \rightarrow \infty$ ), the in-plane modulus initially increases at a rate comparable to that for $h=10 \mu m$ and then increases more rapidly and even exceeds that for $h=10 \mu \mathrm{m}$. Sintering is then arrested and there is no further increase in modulus after about $\sim 100 \mathrm{~h}$. Creep relaxes the in-plane stress and the constraint on the sintering of the vertical and near horizontal cracks. In the absence of Coble creep, the in-plane stress and constraint gradually build-up, first slowing the rate of sintering and then arresting it altogether. Thus, creep of the spats is essential for sintering to proceed. 
1 The characteristic time scales for free asperity sintering and Coble creep relaxation identified

2 by Cocks et al. [11] can be used to help understand the different overall sintering responses.

3 They demonstrated that the characteristic time for free sintering of the asperities is [11]:

4

$$
\tau_{1}=\frac{\left(w_{0} b_{0}\right)^{2}}{8 \Phi_{g}\left(2 \gamma_{s}-\gamma_{g}\right)}
$$

5 and that for Coble creep to fully accommodate the sintering strain is given as:

6

$$
\tau_{2}=\frac{s^{2} \chi h^{3} w_{0}^{2}}{12 R \oplus_{g} b_{0}^{2}\left(2 \gamma_{s}-\gamma_{g}\right)}
$$

7 where $b_{0}$ is the initial asperity contact radius; $w_{0}$ is the initial crack opening and $R$ is the 8 average radius of the splats.

9 For all the cases considered in this study, $\tau_{1}=69.8 \mathrm{~h}$ remains fixed while $\tau_{2}$ varies from $121.7 \mathrm{~h}$

10 for $h=2 \mu \mathrm{m}$ to $3043.6 \mathrm{~h}$ for $h=10 \mu \mathrm{m}$. For $h=2 \mu \mathrm{m}, \tau_{1}$ is comparable to $\tau_{2}$. Therefore, the

11 overall sintering response is determined by both asperity sintering and relaxation by Coble creep. However, for $h=10 \mu m$, asperity sintering is much faster than Coble creep. It is the slowest process that determines the rate of sintering and therefore the overall sintering response is now determined by Coble creep. The factor of 25 increase in relaxation time for these two splat thicknesses (i.e. $\left.\tau_{2}(h=10 \mu m) / \tau_{2}(h=2 \mu m)\right)$ is comparable to a factor of $\sim 11.5$ decrease in the rate of development of the in-plane modulus predicted by the simulation in Figure 11(a). 
(a)

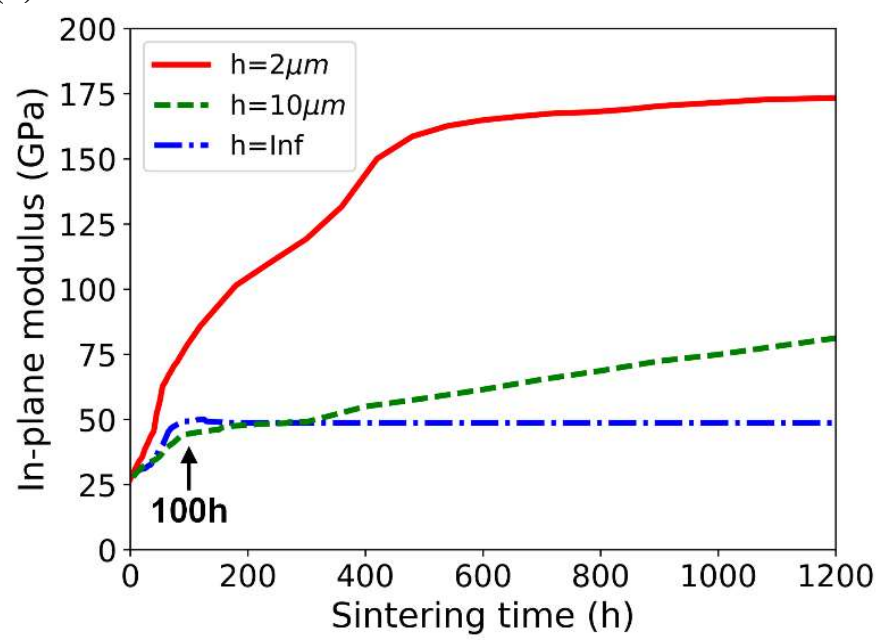

(b)

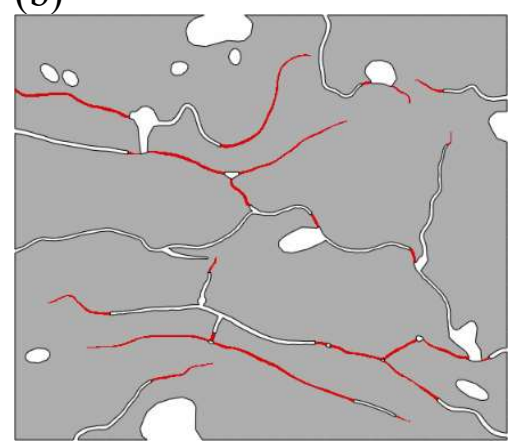

(c)

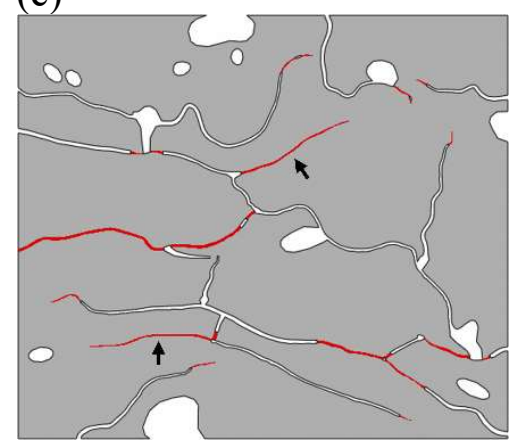

(d)

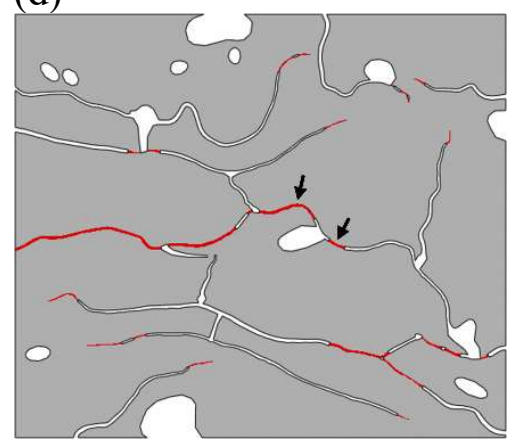

Figure 11 (a) Evolution of the apparent in-plane modulus with sintering time for different YSZ splat thickness and the corresponding microstructure after sintering for $100 \mathrm{~h}$ for (b) $h=2 \mu \mathrm{m}$; (c) $h=10 \mu \mathrm{m}$ and (d) $h=\operatorname{Inf}$. The cracks segments where asperity contact has been established are highlighted in red.

The detailed sintering response also depends on the microstructure, especially during the initial stage of sintering. For a large splat thickness, the initial crack network provides partial relaxation of the constraint on sintering and an initial fast increase of in-plane modulus ensues. As sintering proceeds, cracks close, the sample stiffens and the constraint increases. The torturous nature of the cracks makes relaxation by Coble creep essential for sintering to continue. As a result, sintering eventually stops for $h \rightarrow \infty$ and becomes more gradual for $h=10 \mu \mathrm{m}$ after $\sim 100 \mathrm{~h}$. Moreover, the detailed microstructure evolution also depends on the relative rate of sintering and active relaxation mechanisms. Figure 11 (b)-(d) compare the predicted microstructure for different splat thicknesses after sintering for $100 \mathrm{~h}$. For the thinner splats, more cracks have started healing, although this may not necessarily give rise to a higher 
1 in-plane modulus, which also depends on the sequence at which the cracks have healed. For

2 example, the healing of the crack segment indicated by arrows in Figure 11 (d), partway along

3 a long crack has produced a larger in-plane modulus increase compared with that by healing

4 along short tributary cracks (illustrated in Figure 11 (c)). Healing of the more horizontal cracks

5 is preferred when the constraint of the matrix cannot be readily relaxed by creep (Figure 11 (c-

6 d)) while it becomes more uniform when the matrix is less viscous (Figure 11 (b)).

7

\subsubsection{Effect of grain growth}

The rate of sintering may be slowed by the growth of the columnar intra-splat grains, whose size enters the model through the Coble creep tensor of Eqn (12). Figure 12 shows the effect of varying the grain growth constant $k$ in Eqn (13) on the in-plane modulus evolution. For all cases, the in-plane modulus increases initially at the same rate. Grain growth gradually slows the modulus increase as sintering proceeds.

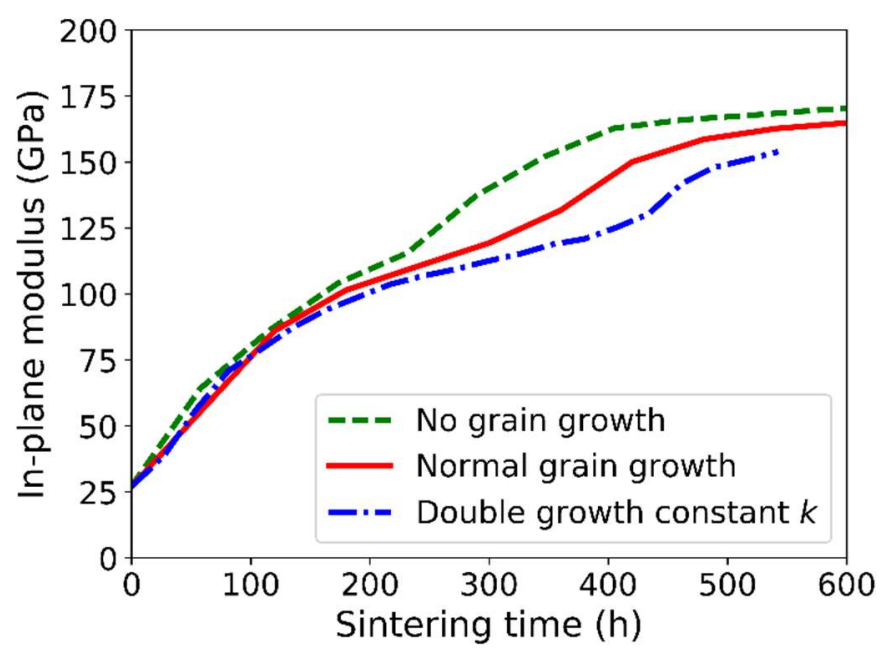

Figure 12 The effect of grain growth on the evolution of the in-plane modulus

If the constraint on sintering from the YSZ splats can be readily relaxed by creep for example when the splat thickness is sufficiently small or the diffusivity for the columnar grain boundaries is much higher than that for the asperity and splat boundaries, the overall sintering response will be controlled by asperity sintering and grain growth has a negligible effect on the sintering response. As the grains increase in size and the rate of Coble creep slows, relaxation 
1 by Coble creep can eventually become the controlling process, resulting in a reduction in the

2 overall sintering rate and the rate of stiffening of the coating, as shown in Figure 12.

3 In a real APS TBC, the growth of the columnar grains can be constrained by grain boundary

4 grooving on the inter-splat crack surfaces [29, 30]. As the grooves deepen, the constraint on

5 grain-growth increases. This suggests that in practical applications, the effect of grain growth

6 on the overall sintering response could be limited.

(a)

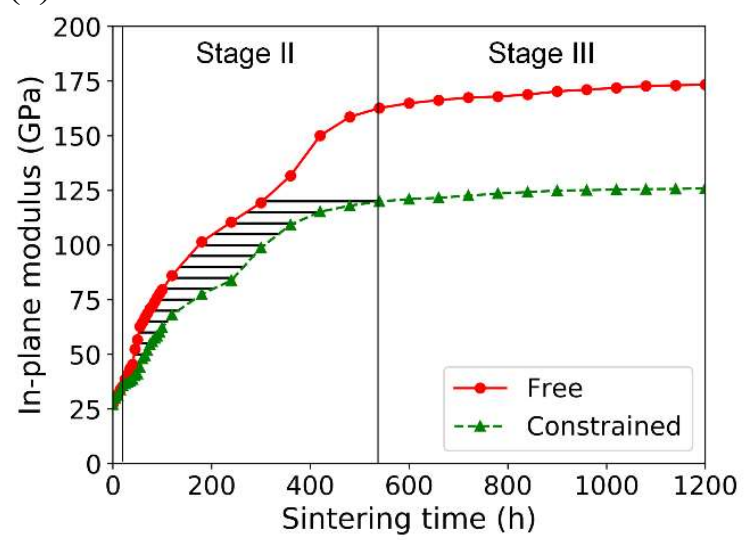

(c)

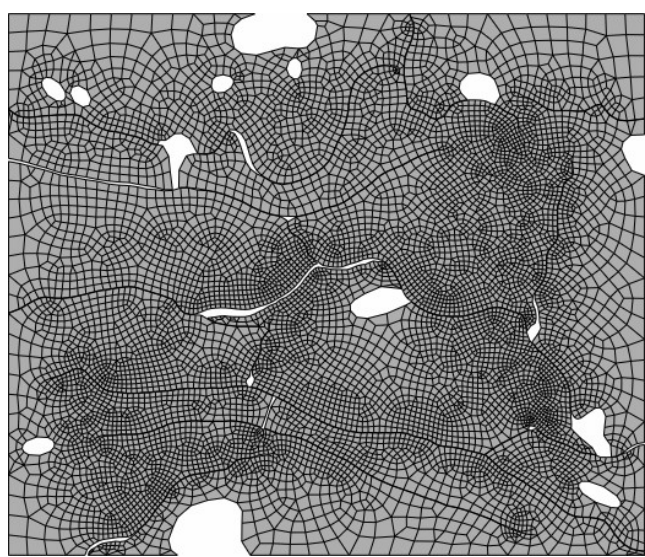

(b)

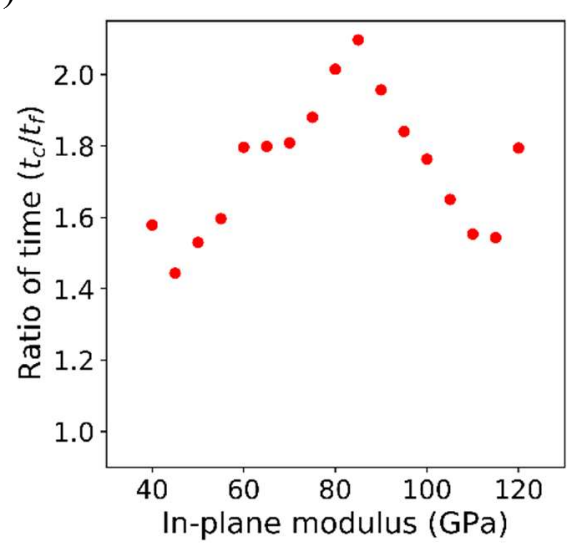

(d)

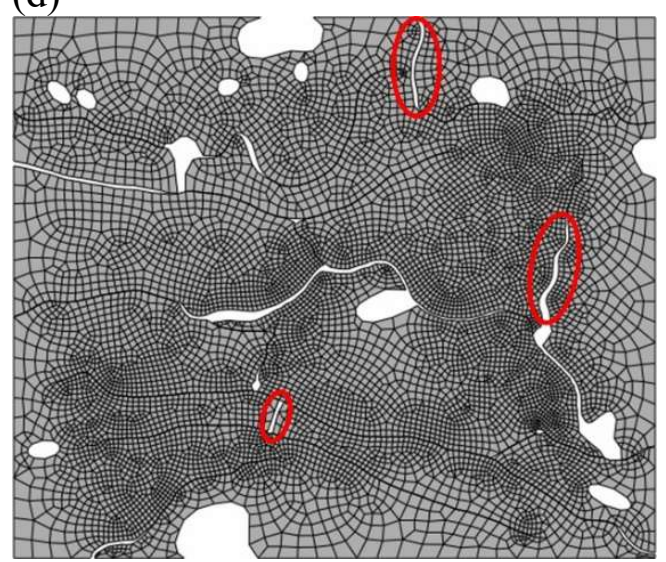

Figure 13 Comparison of the constrained and free sintering: (a) evolution of inplane modulus; (b) the ratio of time to reach a target in-plane modulus and the predicted microstructure after sintering for $1200 \mathrm{~h}$ for (c) free and (d) constrained sintering.

In the above simulations we have concentrated on free sintering of a detached section of coating. In practice the in-plane deformation of the coating is constrained by the substrate. 
1 Constrained sintering has been simulated by fixing the horizontal displacement on the vertical

2 faces of the unit cell, such as that shown in Figure 4(a). The same initial condition as in free sintering is assumed. To start with, the transient heating process is ignored. The resultant inplane modulus evolution is compared with that for free sintering in Figure 13 (a). Three stages are observed in the plot. The two responses are almost identical initially. As sintering proceeds, the constraint begins to slow down the modulus increase (the secondary stage). The retardation effect from the constraint is quantified by plotting the ratio of times to achieve a target in-plane modulus, as shown in Figure 13 (b). This time ratio ranges from 1.4 to 2.1 which is consistent with the prediction from the analytical model of [7]. With prolonged sintering, the in-plane modulus flattens at a lower value (the final stage) for the constrained sintering case. The modelled microstructures at this stage (Figure 13 (c) and (d)) show that the horizontal constraint has prevented the more vertical cracks from closing as highlighted by the red circles in Figure 13 (d). The lower modulus value for constrained sintering at the final stage is due to these residual vertical cracks.

In practice, constrained sintering also includes a transient period leading to the hold temperature during which a thermal expansion mismatch strain $\varepsilon_{T}$ is generated with respect to the substrate. If the $\mathrm{TBC}$ is stress free at a deposition temperature of $\sim 500^{\circ} \mathrm{C}$ [7], an in-plane thermal strain $\varepsilon_{T}=0.4 \%$ is generated when the coating $\left(\alpha=10^{-5} \mathrm{~K}^{-1}\right)$ and the substrate ( $\alpha=1.5 \times 10^{-5} \mathrm{~K}^{-1}$ ) are heated up to an isothermal sintering temperature of $1300{ }^{\circ} \mathrm{C}$, as done in the experiments conducted by Thompson et al. [5]. While existing cracks tend to open to accommodate the thermal strain, the detailed effect is also related to the microstructure. This is explored in Figure 14, where a lateral strain of $0.4 \%$ is applied to the as-deposited microstructure to mimic the heating-up process. 
(a)

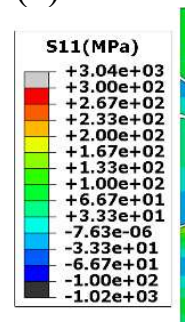

(b)

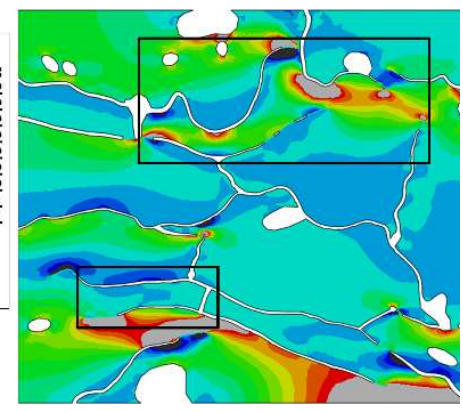

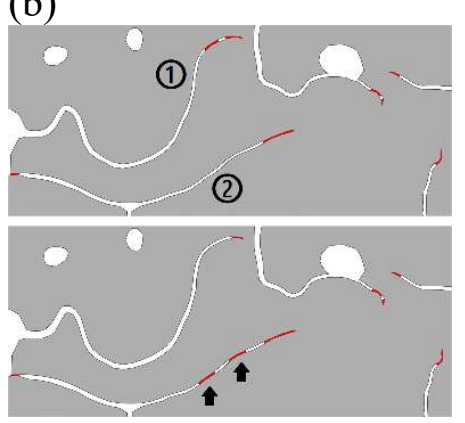

(c)

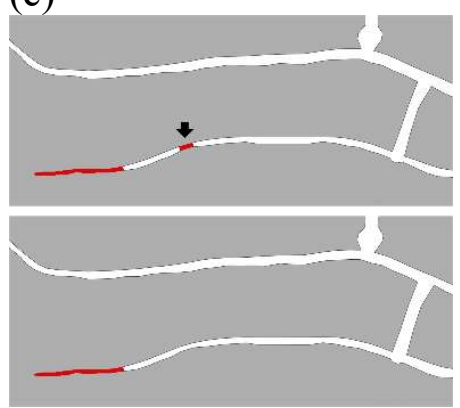

Figure 14 Effect of applying a lateral strain comparable to the thermal strain upon reaching the hold temperature $\varepsilon_{T}=0.4 \%$ : (a) the resultant in-plane stress distribution; (b)-(c) zoom-in images showing the cracks in the boxes in (a) before (upper) and after (lower) applying the thermal expansion mismatch strain. The segments where asperity contact exists are highlighted in red. Both breaking up and establishment of new asperity contacts are observed.

Figure 14 (b) and (c) show that the strain resulting from thermal expansion mismatch can simultaneously de-sinter and separate some asperities, while developing new asperity contacts. For the nearly horizontal cracks, those bridged at one end tend to open, so that asperities separate (e.g. crack (1) of Figure 14 (b)). New asperity contacts can be established locally on the cracks that are bridged at both ends (e.g. crack (2) of Figure 14 (b)). Figure 14 (a) also shows that tensile stresses higher than the strength of the 8YSZ (on the order of 300-500 MPa [23]) is generated and can potentially lead to local fracture. The occurrence of fracture also depends on the thermal history (i.e. heating rate) since this stress relaxes rapidly as a result of sintering and Coble creep as elucidated by Cocks et al. [11]. Still, this tensile stress can open the cracks that are too fine to be included in the FE mesh (see Figure 4 (a)), leading to a lower initial in-plane modulus, as observed experimentally [5].

\subsection{Factors that control the rate of sintering of APS TBCs}

In the previous sections we have evaluated the main factors that control the rate of sintering of an APS TBC. The two main factors that determine the rate of sintering are the rates of sintering of contacting asperities and the rate at which internal stresses can be relaxed by Coble creep of the splats, which depends on the splat thickness and columnar grain size. In section 4.1.2 we 
1 identified two characteristic time scales for sintering of the asperities and creep relaxation. Here

2 we evaluate these effects further and explore how the effect of geometric and material constants on the sintering response can be represented by a sintering map. The rates of these processes change as the coating sinters and it proves instructive to examine the instantaneous rates when constructing a map. This is explored more fully in Appendix A and Supplementary Data S5 where it is demonstrated that the sintering rate is controlled by two non-dimensional indices:

(1) the asperity sintering index $\sqrt{3} \pi^{2} b^{4} / 4 s^{4}$ and (2) the Coble creep relaxation index $\chi h^{3} / G^{2} s^{2}$, where $G$ is a dimensionless geometric factor that is a function of the proportion 9 of the cracks that are bridged by asperities and the aspect ratio of the inter-splat grains, as defined in Eqn (A5) for a single crack and Eqn (S14) for multiple cracks. The map is based on the free sintering of an idealised configuration consisting of an array of preferentially aligned slit cracks (initial average length $2 a$ ) in a transversely isotropic matrix. The cracks are partially bridged by asperities at the tips. The effects of the existence of multiple cracks and the incomplete bridging of the cracks by asperities are lumped into the Coble creep relaxation index through the geometrical factor $G$. Details of the map construction is given in Appendix A. An example of a map using typical material and internal geometric parameters either employed in, or derived from, the simulations is shown in Figure 15. It can be divided into domains (bordered by the dashed lines) where the overall sintering rate is controlled by different kinetic processes. In Domain I, the relaxation rate by Coble creep is much slower than asperity sintering and controls the overall sintering response; in Domain II, the asperity sintering rate is comparable to the Coble creep relaxation rate and the overall sintering response depends on both processes; in Domain III, the asperity sintering rate is much slower than the creep relaxation rate and the overall sintering response is determined by the asperity sintering rate. 


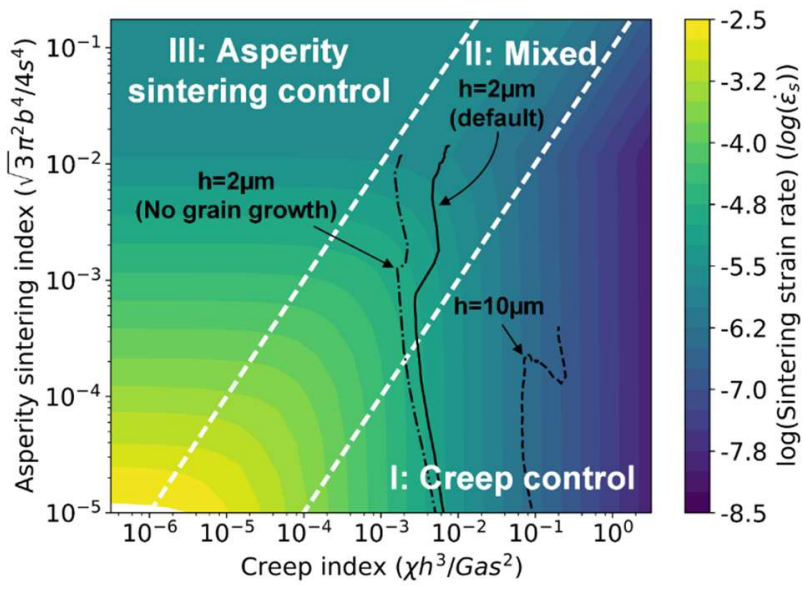

Figure 15 Sintering map summarising the effects of the geometrical parameters. The trajectories obtained from the image-based FE model are superposed on the sintering map showing the rate controlling process at different stages of sintering. A larger splat thickness and grain growth slow down the overall sintering rate and shift the trajectory towards the creep-controlled domain.

Superimposing trajectories obtained from the image-based FE models on the sintering map demonstrates how the rate controlling factor can change as the microstructure evolves. For example, the three black lines in Figure 15 represent results from the image-based model assuming different YSZ splat thickness and grain growth behaviour. For all cases, sintering is initially driven by a limited number of asperities that are in contact. Consequently, the creep relaxation rate is low and controls the overall sintering rate at the beginning. As sintering proceeds, more asperities are brought into contact leading to (1) an increased average asperity contact area (and therefore an increase of the asperity sintering index) and (2) a higher driving force for creep relaxation due to the increasing number of contacting asperities. This leads to a decrease in the creep relaxation index as reflected by the curves inclining to the left during the early stages of sintering. For $\mathrm{h}=2 \mu \mathrm{m}$, the creep relaxation rate becomes comparable to the asperity sintering rate as an increasing portion or the cracks become bridged by asperities and the overall sintering rate depends on both processes. Full sintering occurs gradually leading to a decrease in the average crack length $a$. This reduces the overall compliance of the coating and therefore the creep relaxation rate. With prolonged sintering, the growth of the columnar 
1 grains also retards creep relaxation, leading to a higher creep index. For $\mathrm{h}=10 \mu \mathrm{m}$, the creep

2 relaxation rate remains low and controls the overall sintering rate throughout the simulation

3

4 It is expected that the creep relaxation rate will become the rate controlling process as the period. coating approaches full sintering ( $a$ approaches zero). However, this only occurs after a time much longer than the typical lifetime of the APS TBC. Therefore, the simulation has been interrupted before this limit is reached.

Maps of this type, together with the characteristic timescales identified in section 4.1.2, allow the effects of the microstructure developed during deposition of the coating on the sintering response to be evaluated. Increasing the splat thickness and size of the intra-splat grains slows the creep rate and can be a practical way of controlling the microstructure to develop a more sintering-resistant APS TBC. Also, decreasing the effective boundary diffusivity (e.g. via doping [31]) is always beneficial to increasing the coating's sintering resistance.

\section{Conclusions}

An image-based micromechanical model has been developed for the sintering of APS TBCs.

A constitutive law has been developed for the inter-splat cracks using a thermodynamic variational principle. This together with an anisotropic creep law for the YSZ splats and an ideal grain growth law constitutes the framework of the proposed sintering model, which was then implemented using microstructurally realistic meshes representative of an as-deposited APS TBC. The following conclusions can be drawn from experimental observations and model results:

(1) Sintering occurs in the form of sequential healing of the inter-splat cracks, which is captured by the proposed model. Moreover, the interaction of adjacent cracks during the sintering proposes is observed in the model. This interaction can be quite subtle - 
in systems with a high creep resistance (large splats and large intra-splat grains) the healing of some cracks is accommodated by the opening of others. The validity of the model is confirmed by experimental results for the crack density distribution and inplane modulus evolution;

(2) The model enables a correlation between the modulus evolution and the sinter-induced microstructure changes. The wavy splat structure results in a significant part of the coating being under bending when loaded. The vertical intra-splat cracks unload the sample locally. Both factors contribute to the significantly low in-plane modulus for the as-deposited TBC. Sintering increases the in-plane modulus by 'pinning' the adjacent splats so that a larger proportion of the coating deforms by stretching. Moreover, the region that experiences unloading adjacent to the vertical cracks is diminished as a result of sintering;

(3) The sensitivity of the model results to the geometric parameters and sintering condition (e.g. free vs constrained) is explored. The physical reasons for the sensitivity are interpreted with the help of the simulated microstructures. The model sensitivity is summarised in the form of a sintering map where the rate controlling process is identified for different combinations of the geometric parameters. Moreover, the link between the internal geometric parameters and the experimentally measurable microstructural features is discussed. Based on this, a calibration routine is suggested;

(4) For real application purposes, our model results imply that microstructure engineering should focus on increasing the splat thickness and columnar grain size and introducing more vertical cracks to create APS TBCs with improved sintering resistance. 
1 The model framework can be potentially extended to 3D micrographs. The current

2 development of Xe Plasma-FIB [32] and femtosecond laser ablation in situ SEM [33] may

3 provide the essential 3D microstructural information of an APS TBC. This together with the

4 growth of computing power will enable the 3D image-based sintering models to be developed

5 that can provide more realistic and detailed prediction on the sintering response. This will be

6 the focus of future work.

\section{Acknowledgement}

8 This work was supported by Mitsubishi Heavy Industries (MHI), Japan. XZ is grateful for the 9 help on the user element development from Dr. Elsiddig Elmukashfi. 


\section{References}

[1] N.P. Padture, M. Gell, E.H. Jordan, Materials science - Thermal barrier coatings for gas-turbine engine applications, Science 296(5566) (2002) 280-284.

[2] S. Paul, Assessing Coating Reliability Through Pore Architecture Evaluation, J Therm Spray Techn 19(4) (2010) 779-786.

[3] A. Guignard, G. Mauer, R. Vassen, D. Stover, Deposition and Characteristics of SubmicrometerStructured Thermal Barrier Coatings by Suspension Plasma Spraying, J Therm Spray Techn 21(3-4) (2012) 416-424.

[4] S.R. Choi, D.M. Zhu, R.A. Miller, Effect of sintering on mechanical properties of plasma-sprayed zirconia-based thermal barrier coatings, J Am Ceram Soc 88(10) (2005) 2859-2867.

[5] J.A. Thompson, T.W. Clyne, The effect of heat treatment on the stiffness of zirconia top coats in plasma-sprayed TBCs, Acta Mater 49(9) (2001) 1565-1575.

[6] T. Wakui, J. Malzbender, R.W. Steinbrech, Strain dependent stiffness of plasma sprayed thermal barrier coatings, Surf Coat Tech 200(16-17) (2006) 4995-5002.

[7] A.C.F. Cocks, N.A. Fleck, Constrained sintering of an air-plasma-sprayed thermal barrier coating, Acta Mater 58(12) (2010) 4233-4244.

[8] D.M. Zhu, R.A. Miller, Thermal conductivity and elastic modulus evolution of thermal barrier coatings under high heat flux conditions, J Therm Spray Techn 9(2) (2000) 175-180.

[9] N.A. Fleck, A.C.F. Cocks, S. Lampenscherf, Thermal shock resistance of air plasma sprayed thermal barrier coatings, J Eur Ceram Soc 34(11) (2014) 2687-2694.

[10] X. Zhang, J. Kulczyk-Malecka, J. Carr, P. Xiao, P.J. Withers, 3D characterization of porosity in an air plasma-sprayed thermal barrier coating and its effect on thermal conductivity, J Am Ceram Soc 101(6) (2018) 2482-2492.

[11] A. Cocks, N. Fleck, S. Lampenscherf, A brick model for asperity sintering and creep of APS TBCs, J Mech Phys Solids 63 (2014) 412-431.

[12] N.A. Fleck, A.C.F. Cocks, A multi-scale constitutive model for the sintering of an air-plasmasprayed thermal barrier coating, and its response, under hot isostatic pressing, J Mech Phys Solids 57(4) (2009) 689-705.

[13] A. Cipitria, I.O. Golosnoy, T.W. Clyne, A sintering model for plasma-sprayed zirconia TBCs. Part I: Free-standing coatings, Acta Mater 57(4) (2009) 980-992. 
1 [14] A. Cipitria, I.O. Golosnoy, T.W. Clyne, A sintering model for plasma-sprayed zirconia thermal

2 barrier coatings. Part II: Coatings bonded to a rigid substrate, Acta Mater 57(4) (2009) 993-1003.

3 [15] B. Lv, X. Fan, H. Xie, T.J. Wang, Effect of neck formation on the sintering of air-plasma-sprayed 4 thermal barrier coating system, J Eur Ceram Soc 37(2) (2017) 811-821.

5 [16] A.C.F. Cocks, S.P.A. Gill, J.Z. Pan, Modeling microstructure evolution in engineering materials, $6 \quad$ Adv Appl Mech 36 (1999) 81-162.

7 [17] N. Balasubramanian, T.G. Langdon, Flow processes in superplastic yttria-stabilized zirconia: A 8 Deformation Limit Diagram, Mat Sci Eng a-Struct 409(1-2) (2005) 46-51.

9 [18] S. Kumar, A.C.F. Cocks, Computational modelling of constrained sintering in EB-PVD thermal 10 barrier coatings, Model Simul Mater Sc 21(6) (2013).

11 [19] K. Shanmugam, Multiscale modelling of sintering in thermal barrier coatings, University of 12 Oxford, 2010.

[20] G.R. Li, H. Xie, G.J. Yang, G. Liu, C.X. Li, C.J. Li, A comprehensive sintering mechanism for TBCs-Part I: An overall evolution with two-stage kinetics, J Am Ceram Soc 100(5) (2017) 2176-2189. [21] J. Pan, A.C.F. Cocks, S. Kucherenko, Finite element formulation of coupled grain-boundary and surface diffusion with grain-boundary migration, P Roy Soc a-Math Phy 453(1965) (1997) 2161-2184. [22] S. Swaroop, M. Kilo, C. Argirusis, G. Borchardt, A.H. Chokshi, Lattice and grain boundary diffusion of cations in 3 YTZ analyzed using SIMS, Acta Mater 53(19) (2005) 4975-4985.

[23] X.R. Ren, W. Pan, Mechanical properties of high-temperature-degraded yttria-stabilized zirconia, Acta Mater 69 (2014) 397-406.

[24] A.M. Brown, M.F. Ashby, Correlations for Diffusion Constants, Acta Metall Mater 28(8) (1980) 1085-1101.

[25] A.H. Chokshi, Diffusion, diffusion creep and grain growth characteristics of nanocrystalline and fine-grained monoclinic, tetragonal and cubic zirconia, Scripta Mater 48(6) (2003) 791-796. [26] B.W. Lv, R. Mucke, X.L. Fan, T.J. Wang, O. Guillon, R. Vassen, Sintering resistance of advanced plasma-sprayed thermal barrier coatings with strain-tolerant microstructures, J Eur Ceram Soc 38(15) (2018) 5092-5100.

[27] M. Shinozaki, T.W. Clyne, A methodology, based on sintering-induced stiffening, for prediction of the spallation lifetime of plasma-sprayed coatings, Acta Mater 61(2) (2013) 579-588. 
1 [28] S. Paul, A. Cipitria, I.O. Golosnoy, L. Xie, M.R. Dorfman, T.W. Clyne, Effects of impurity content 2 on the sintering characteristics of plasma-sprayed zirconia, J Therm Spray Techn 16(5-6) (2007) 7983803.

4 [29] W.W. Mullins, The Effect of Thermal Grooving on Grain Boundary Motion, Acta Metall Mater 5 6(6) (1958) 414-427.

6 [30] K.A. Erk, C. Deschaseaux, R.W. Trice, Grain-boundary grooving of plasma-sprayed yttria7 stabilized zirconia thermal barrier coatings, J Am Ceram Soc 89(5) (2006) 1673-1678.

8 [31] M. Matsumoto, N. Yamaguchi, H. Matsubara, Low thermal conductivity and high temperature 9 stability of ZrO2-Y2O3-La2O3 coatings produced by electron beam PVD, Scripta Mater 50(6) (2004) $10 \quad 867-871$.

11 [32] T.L. Burnett, R. Kelley, B. Winiarski, L. Contreras, M. Daly, A. Gholinia, M.G. Burke, P.J. 12 Withers, Large volume serial section tomography by Xe Plasma FIB dual beam microscopy, Ultramicroscopy 161 (2016) 119-129.

14 [33] M.P. Echlin, M. Straw, S. Randolph, J. Filevich, T.M. Pollock, The TriBeam system: Femtosecond laser ablation in situ SEM, Mater Charact 100 (2015) 1-12.

16 [34] M. Kachanov, B. Shafiro, I. Tsukrov, A crack in an infinite anisotropic two-dimensional solid, 17 Handbook of Elasticity Solutions, Springer Netherlands, Dordrecht, 2003, pp. 131-154. 


\section{Appendix A. The sintering map}

2 In section 4 we demonstrate that sintering is dominated by healing of the nearly horizontal

3 wavy cracks. Here we adopt a simplified description of this process, which enables us to derive

4 simple analytical relationships for the sintering process. It proves instructive to first consider a

5 simple example where a single horizontal opened crack with length $2 a$ is embedded in an

6 infinitely large plate. The plate undergoes transversely isotropic Coble creep characterised by

7 Eqn. (S8). Assume that the crack normal coincides with the global z-axis. We further assume

8 that the crack surfaces are partially bridged by asperities at the tips with the total length of 9 unbridged segment equal to $2 l$ as shown in Figure A-1.

10

11

12

13

$$
\dot{w}_{n}=-T_{n} \frac{3 \Phi_{g} \pi a}{\chi h^{3}}\left(3+\sqrt{\frac{2\left(1+\chi^{2}\right)}{\chi^{2}}}\right)^{1 / 2} g\left(\frac{a}{l}\right)
$$

17 where $g\left(\frac{a}{l}\right)$ is a geometric factor that depends on the ratio of the total crack length compared

Figure A-1 Schematics of one crack with the tips bridged by asperities.

Compatibility dedicates that the crack closure rate due to sintering is equal to that by creep.

Consider a uniform normal traction $T_{n}$ on the bridged crack segments and assume $\chi \ll 1$ and that the crack remains elliptical, the average crack closure rate by matrix creep can be calculated as [34]:

to the un-bridged crack length: 


$$
g\left(\frac{a}{l}\right)=\left\{1-\frac{2 l}{\pi a} \ln \left[\sqrt{\left(\frac{a}{l}\right)^{2}-1}+\frac{a}{l}\right]-\frac{2}{\pi} \cot ^{-1}\left(\sqrt{\left(\frac{a}{l}\right)^{2}-1}\right)\right\}
$$

2 According to Eqn (8), the crack closure rate by asperity sintering is:

4 Both asperity sintering and Coble creep of the YSZ splats involve boundary diffusion.

Assuming the same effective boundary diffusivity at the asperity and inter-splat grain scales, equating Eqn (A1) and Eqn (A3) gives:

$$
\dot{w}_{n}=-\frac{3 \Phi_{g} \pi}{s^{2}} \frac{T_{s}}{\left(\sqrt{3} \pi^{2} b^{4} / 4 s^{4}\right)+\left(\chi h^{3} / G a s^{2}\right)}
$$

where

$$
G=\left(3+\sqrt{\frac{2\left(1+\chi^{2}\right)}{\chi^{2}}}\right)^{1 / 2} g\left(\frac{a}{l}\right)
$$

This dimensionless factor arises due to (1) the anisotropic (transversely isotropic) Creep response of the matrix material and (2) partial bridging of the crack by asperities.

Eqn (A4) shows that the sintering rate is controlled by two non-dimensional indices: (1) the asperity sintering index $\sqrt{3} \pi^{2} b^{4} / 4 s^{4}$ and (2) the Coble creep relaxation index $\chi h^{3} / G a s^{2}$. These indices form the axes of the sintering map in section 4.3. The larger the indices, the slower the corresponding process will be. It is the larger of these two indices that determines the overall sintering rate. For example, as the asperity grows (i.e. increasing $b$ ), the diffusion distance increases and the asperity sintering rate decreases. Similarly, a larger splat thickness $h$ or columnar grain size will increase the diffusion distance for Coble creep thus reducing the creep relaxation rate. The effects of anisotropic viscosity and the incomplete bridging of the crack are lumped into the Coble creep index through the dimensionless geometric factor $G$.

$$
\dot{w}_{n}=\frac{8 \oplus_{g}\left[T_{n} B(b)-n_{a} A(b)\right]}{n_{a} \pi b^{4} B(b)}
$$




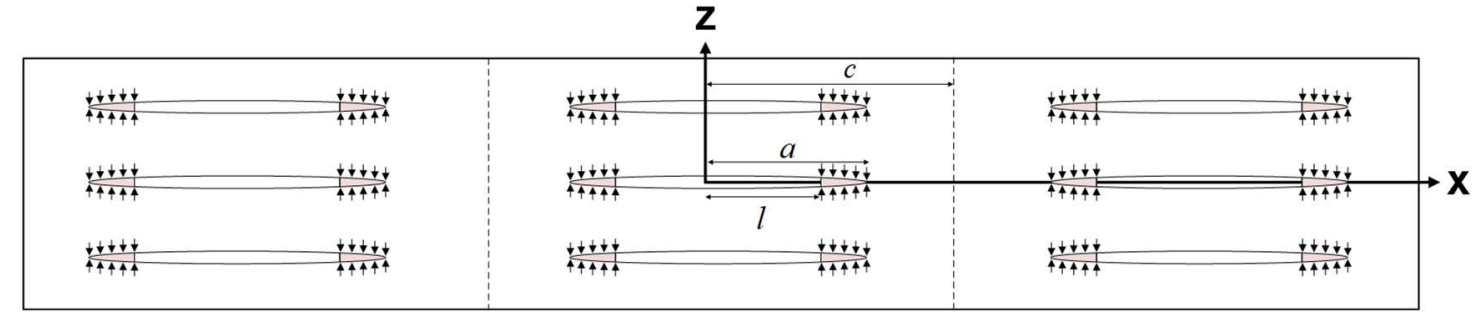

Figure A-2 Idealisation of the topcoat as a solid containing aligned slit cracks. The matrix creeps in a transversely isotropic manner. The overall response of the sloid remains transversely isotropic with additional compliance from the cracks. The sintering force pulls the crack surfaces against each other.

The inter-splat cracks within an APS TBC can be better represented by an array of colinear cracks with initial length $2 a$ that are spaced by a distance $2 c$ as shown in Figure A-2. The solution for the average crack closure rate has the same form as Eqn (A4) except that $G$ now has an additional contribution from the increased compliance due to the existence of multiple cracks. The detailed expressions are given in Supplementary Data S5.

In order to superimpose the results from the computational sintering models on the sintering map, we adopt $a=50 \mu m$ and $c=150 \mu m$ for the cracked solid to represent the as-deposited TBC. The instantaneous asperity contact radius is output from the FE model at different instants. Based on the local asperity contact radius, the crack segments can be divided into three categories: (i) inactive segments where full sintering has been achieved (i.e. $b=s / 2$ ); (ii) active and free segments where no asperity contact has been established (i.e. $b=0$ ) and (iii) active and sintering segments where asperities are in contact but have not fully sintered. The effective crack length $a$ is updated at each instant by scaling the initial value (i.e. $50 \mu m$ ) with the ratio of total length of the active crack segments (These include both un-bridged and bridged and sintering crack segments i.e. Category ii and iii) over the total length of the initial crack segments. Similarly, the ratio of the length of bridged and sintering crack segments (Category iii) over the length of the active crack segment (Category ii and iii) is computed. The effective crack length $a$; the ratio of the bridged and sintering crack length over the total active 
1 crack length and the diameter of the columnar grains are used to determine the geometrical

2 factor $G$ numerically which is then used to calculate the Coble creep relaxation index

$3 \chi h^{3} / G_{a s}^{2}$. The average contact radius is computed from the model output and used to

4 determine the asperity sintering index $\sqrt{3} \pi^{2} b^{4} / 4 s^{4}$. Throughout a sintering simulation, the

$5 \quad$ splat thickness $h$ and crack spacing $c$ are kept constant. 


\section{Figure Captions}

Figure 1 SEM micrographs taken from the polished cross-sections (top row) and fracture surfaces (bottom row) of the APS TBC sample showing (a) curved YSZ splats with mainly horizontal inter-splat cracks in the as-deposited sample; (b) partially healed inter-splat cracks after sintering for $8 \mathrm{hrs}$ at $1400^{\circ} \mathrm{C}$; (c) the curved YSZ splats containing columnar grains with sharp grain boundaries and (d) YSZ splats that become roughened (e.g. the region in the ellipse) and the intra-splat columnar grains that become more spherical after sintering (e.g. the region in the square).

Figure 2 SEM micrographs showing the main microstructural features that evolve during sintering of an APS TBC.

Figure 3 The inter-splat cracks with spherical cap asperities of base radius $R_{0}$ on the crack surfaces. The centre-to-centre distance between the asperities is $s$. The crack opening is $w$. When the opening is larger than the initial asperity height, the crack surfaces are free to displace with respect to each other (upper figure). Cracks are in contact where the opening is smaller than the asperity height e.g. adjacent to a crack tip (lower figure). Sintering leads to an increase in the contact area and a closure of the crack.

Figure 4 FE mesh generation from the cross-sectional micrographs LHS: SEM micrographs and RHS: the FE meshes developed from the corresponding micrographs.

Figure 5 Evolution of the elastic modulus as a function of sintering time at $1300^{\circ} \mathrm{C}$ : experimental data after [5] and the model results for three representative areas. The vertical error bars on the average curve represent the standard deviation of results from the three meshes.

Figure 6 The microstructure of one representative area and how it evolves during sintering: (a) as deposited, where the yellow arrows indicate the crack segments that are initially in contact; (b) after sintering for $50 \mathrm{hrs}$ - the green arrows indicate a crack which is initially open, its faces are brought into contact and they start healing as a result of sintering elsewhere, while the red arrows highlight a crack that has opened more up to this sintering time; (c) after sintering for $75 \mathrm{hrs}$ and (d) after sintering for $100 \mathrm{hrs}$ - the yellow ellipses indicate the almost vertical cracks that have remained open.

Figure 7 Quantitative analysis of the crack orientation: micrographs showing the region of interest in (a) as-deposited and (b) as-sintered $\left(1400^{\circ} \mathrm{C} 8 \mathrm{hrs}\right)$ samples, from which the crack segments have been analysed - (b) also shows the coordinate system used for the orientation 
definition; (c) the total crack length as a function of the angle between the coating's in-plane direction and the crack segment normal ( $\theta$ as defined in (b)). The black arrows highlight the most significant length reduction.

Figure 8 Sintering-induced partial healing of one inter-splat crack: (a) FE model before sintering; (b) after sintering for $8 \mathrm{hrs}$ at $1400^{\circ} \mathrm{C}$, where the user elements that initially represent the crack and have fully sintered merge into the YSZ continuum and (c) SEM cross-sectional micrograph showing one inter-splat crack with a similar morphology to (a), which has become partially healed after sintering.

Figure 9 Model prediction of the evolution of the apparent in-plane modulus as a result of sintering at $1300^{\circ} \mathrm{C}$ and the corresponding microstructure at different times, indicated by the arrows in (a) - in-plane stress (S11) contours are superimposed on the microstructure plots (b) to (d) when a surface traction of $2 \mathrm{MPa}$ is applied to the right edge in the $\mathrm{x}$-direction.

Figure 10 Evolution of the apparent in-plane modulus for different asperity spacings (a) and the corresponding microstructure predicted at different stages of sintering as indicated by the arrows for (b) $s=2.5 R_{0}$ and (c) $s=10 R_{0}$. The black arrows indicate the crack segments that have healed while the red arrows indicate the crack segments that either open or do not sinter.

Figure 11 (a) Evolution of the apparent in-plane modulus with sintering time for different YSZ splat thickness and the corresponding microstructure after sintering for $100 \mathrm{~h}$ for (b) $h=2 \mu \mathrm{m}$ ; (c) $h=10 \mu \mathrm{m}$ and (d) $h=\mathrm{Inf}$. The cracks segments where asperity contact has been established are highlighted in red.

Figure 12 The effect of grain growth on the evolution of the in-plane modulus

Figure 13 Comparison of the constrained and free sintering: (a) evolution of in-plane modulus; (b) the ratio of time to reach a target in-plane modulus and the predicted microstructure after sintering for $1200 \mathrm{~h}$ for $(\mathrm{c})$ free and (d) constrained sintering.

Figure 14 Effect of applying a lateral strain comparable to the thermal strain upon reaching the hold temperature $\varepsilon_{T}=0.4 \%$ : (a) the resultant in-plane stress distribution; (b)-(c) zoom-in images showing the cracks in the boxes in (a) before (upper) and after (lower) applying the thermal expansion mismatch strain. The segments where asperity contact exists are highlighted in red. Both breaking up and establishment of new asperity contacts are observed. 
2 Figure 15 Sintering map summarising the effects of the geometrical parameters. The 3 trajectories obtained from the image-based FE model are superposed on the sintering map 4 showing the rate controlling process at different stages of sintering. A larger splat thickness 5 and grain growth slow down the overall sintering rate and shift the trajectory towards the creep6 controlled domain.

7

\section{Table Captions}

9

10 Table 1 Default geometrical and material constants used in the FE model. 


\section{Supplementary Information: An image-based model for the sintering of air plasma sprayed thermal barrier coatings}

Xun Zhang ${ }^{1,}{ }^{*}$, Alan C.F. Cocks ${ }^{1}$, Yoshifumi Okajima ${ }^{2}$, Kazuma Takeno ${ }^{2}$, Taiji Torigoe ${ }^{2}$

${ }^{1}$ Department of Engineering Science, University of Oxford, Parks Road, Oxford OX1 3PJ, UK

${ }^{2}$ Mitsubishi Heavy Industries, Ltd. 1-1 Shinhama, 2-chome, Arai-cho, Takasago, Hyogo, 6768686, Japan

\section{Determination of the rate function for the asperity contact radius}

We assume the axisymmetric contact geometry as shown in Figure S-1. It is convenient to define a reference configuration as illustrated in Figure S-1 (a). In the reference configuration, the asperity is simplified as a spherical cap of radius of curvature $R_{1}$ with a base radius of $R_{0}$. The volume of the asperity reads:

$$
V_{\text {cap }}=\frac{\pi}{3} \frac{R_{0}^{3}}{\sin ^{3} \theta}(2+\cos \theta)(1-\cos \theta)^{2}
$$

As demonstrated by Cocks et al. [1], for the sintering of arrays of spherical particles, the radius of the spherical particle remains essentially constant during the early stages of sintering and the evolving shape can be solely described by the contact radius $b$. Therefore, In the early stage of asperity sintering, the asperity is represented by a truncated spherical cap with a base radius $R_{0}$ connected to a cylindrical region with a base radius $b$ and height $h$ (Figure A-1(b)). As the contact grows during sintering, the size of the spherical cap reduces, until it is consumed by the cylindrical region when $b=R_{0}$. As the asperity evolves, volume conservation dictates:

$$
h= \begin{cases}\frac{2}{3} \frac{R_{0}^{3}}{2 b^{2} \sin ^{3} \theta}\left[1-0.5 \sqrt{1-\left(\frac{b \sin \theta}{R_{0}}\right)^{2}\left(2+\left(\frac{b \sin \theta}{R_{0}}\right)^{2}\right)}\right] & \text { if } b<R_{0} \\ \frac{R_{0}^{3}}{3 b^{2} \sin ^{3} \theta}\left(\cos ^{3} \theta-3 \cos \theta+2\right) & \text { if } b \geq R_{0}\end{cases}
$$

As a result, the opening of the crack follows as: 
$w=\left\{\begin{array}{lr}\frac{2}{3} \frac{R_{0}^{3}}{2 b^{2} \sin ^{3} \theta}\left[1-0.5 \sqrt{1-\left(\frac{b \sin \theta}{R_{0}}\right)^{2}\left(2+\left(\frac{b \sin \theta}{R_{0}}\right)^{2}\right)}\right]+\frac{R_{0}^{2}}{\sin ^{2} \theta} \sqrt{1-\left(\frac{b \sin \theta}{R_{0}}\right)^{2}}-\frac{R_{0}}{\tan \theta} & \text { if } b<R_{0} \\ \frac{R_{0}^{3}}{3 b^{2} \sin ^{3} \theta}\left(\cos ^{3} \theta-3 \cos \theta+2\right) & \text { if } b \geq R_{0}\end{array}\right.$

(a)

(b)
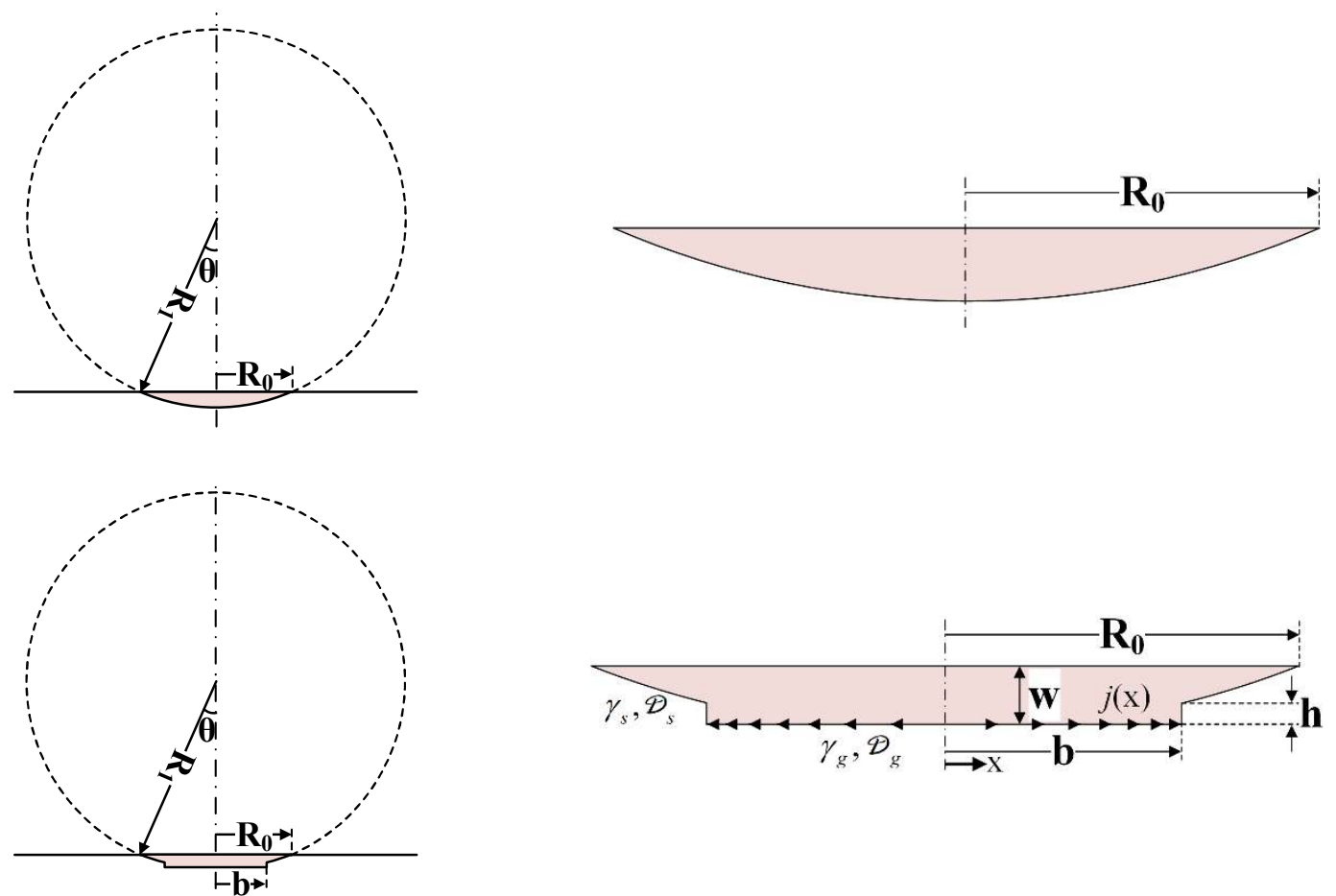

(c)
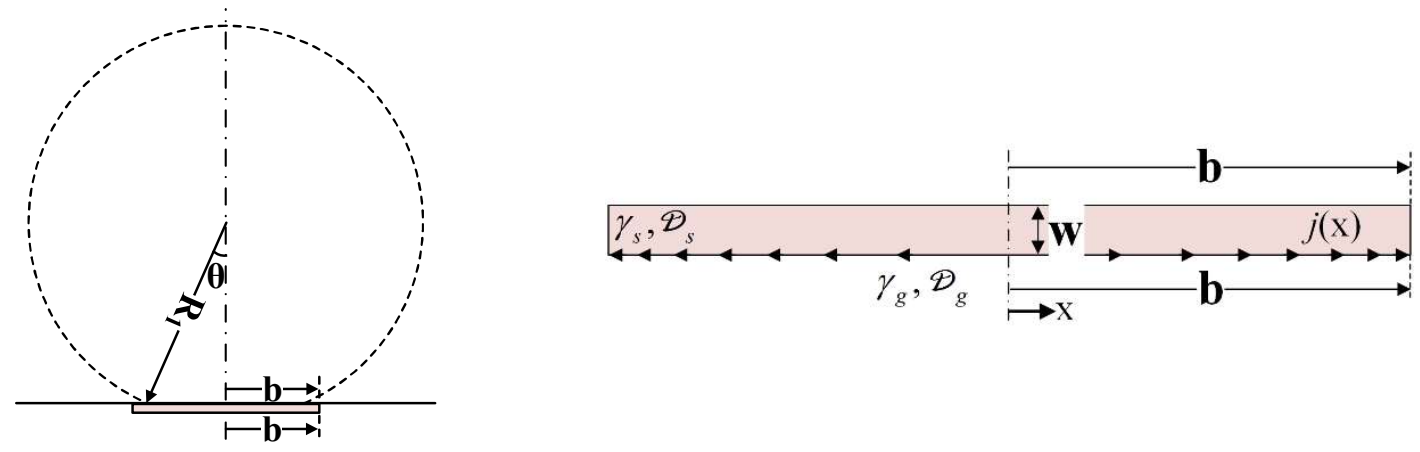

Figure S-1 Schematics showing one asperity: (a) the reference configuration where the asperity is not in contact; (b) the early stage of asperity sintering when the asperity is simplified as a truncated spherical cap with a base radius $R_{0}$ connected to a cylindrical region of radius $b$ and (c) after prolonged sintering when the asperity turns into a cylinder. The definition of fluxes, surface and interface energies and diffusivities are shown on the magnified asperity in (b) and (c). 
The Gibbs free energy per unit volume is given by Eqn (2). The rate of change of Gibbs free energy reads:

$$
\dot{G}=\frac{\partial G}{\partial b} \dot{b}-T_{n} \frac{\partial w}{\partial b} \dot{b}=\left[n_{a} A(b)-T_{n} B(b)\right] \dot{b}
$$

where

$$
\begin{aligned}
& A(b)= \begin{cases}2 \pi\left\{\gamma_{g} b-\gamma_{s} b-\frac{\gamma_{s} b R_{0}}{\sqrt{R_{0}^{2}-b^{2} \sin ^{2} \theta}}+\gamma_{s} \frac{2 R_{0}^{4}-2 R_{0}^{3} \sqrt{R_{0}^{2}-b^{2} \sin ^{2} \theta}-R_{0}^{2} b^{2} \sin ^{2} \theta+2 b^{4} \sin ^{4} \theta}{3 b^{2} \sin ^{3} \theta \sqrt{R_{0}^{2}-b^{2} \sin ^{2} \theta}}\right\} & \text { if } b<R_{0} \\
2 \pi b\left(\gamma_{g}-2 \gamma_{s}\right)-\frac{2 \pi R_{0}^{3}}{3 b^{2} \sin ^{3} \theta}\left(\cos ^{3} \theta-3 \cos \theta+2\right) \gamma_{s} & \text { if } b \geq R_{0}\end{cases} \\
& B(b)= \begin{cases}\frac{2 R_{0}^{2}}{3 b \sin ^{2} \theta \sqrt{R_{0}^{2} / \sin ^{2} \theta-b^{2}}}\left[\frac{2 R_{0}^{2}}{b^{2} \sin ^{2} \theta}-1-\frac{2 R_{0}}{b^{2} \sin \theta} \sqrt{R_{0}^{2} / \sin ^{2} \theta-b^{2}}-\frac{b^{2} \sin ^{2} \theta}{R_{0}^{2}}\right] & \text { if } b<R_{0} \\
-\frac{2 R_{0}^{3}}{3 b^{3} \sin ^{3} \theta}\left(\cos ^{3} \theta-3 \cos \theta+2\right) & \text { if } b \geq R_{0}\end{cases}
\end{aligned}
$$

Substituting Eqn (5) into Eqn (3) gives:

$$
\psi_{a}=\frac{\pi b^{4} \dot{w}^{2}}{16 \Phi_{g}}=\frac{\pi b^{4} \dot{b}^{2}}{16 \Phi_{g}} B(b)^{2}
$$

and $\delta(\dot{G}+\psi)=0$ gives Eqn (8).

\section{The Coble creep tensor}

Following Greenwood [2], Cocks et al. [3] idealised the columnar grains as ellipsoids with the long axis aligned with the direction normal to the splat surface (local z-direction). Eqn (12) then reads using a Voigt notation: 


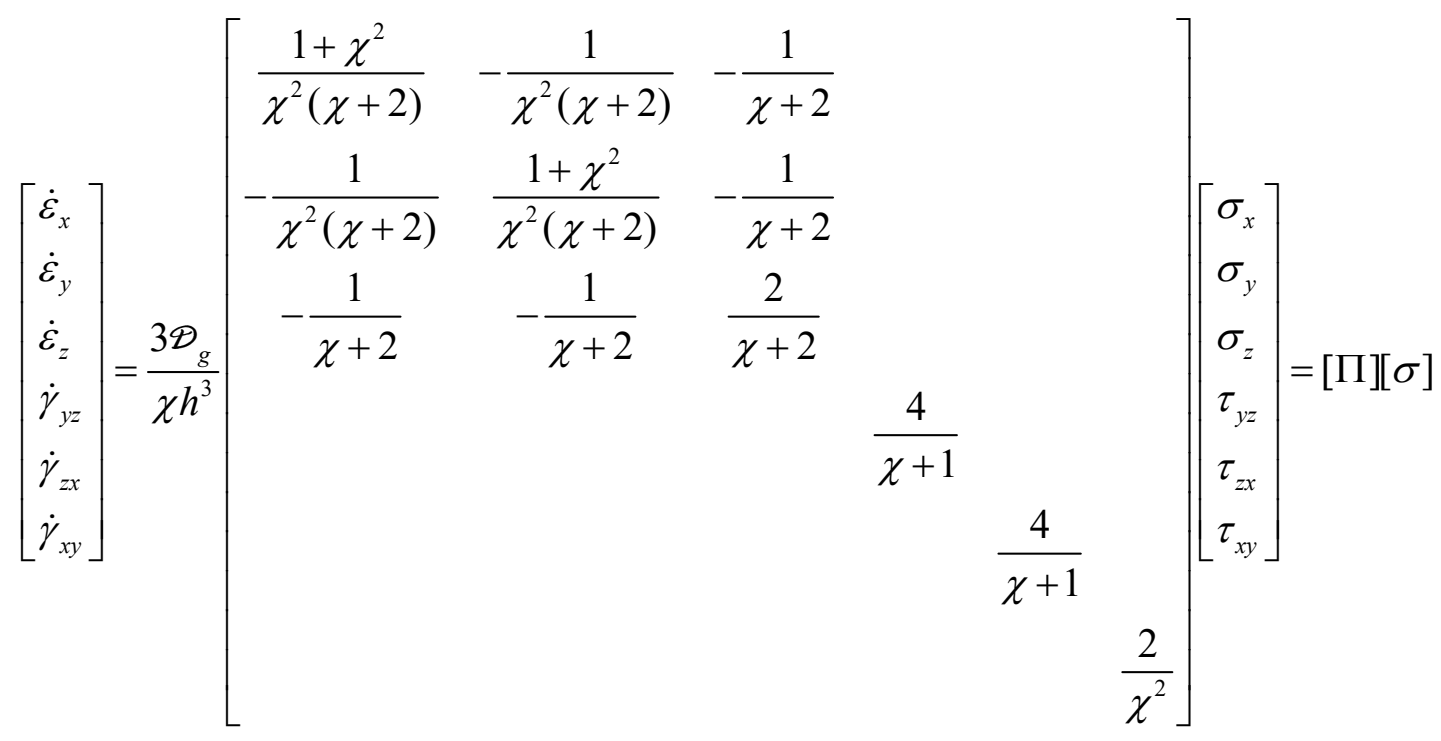

where $\chi=d / 2 h$ is the aspect ratio of the columnar grains.

\section{Sintering test and modulus measurement}

\subsection{Sintering test}

TBC samples $1 \mathrm{~mm}$ in thickness that have been applied by APS on a $5 \mathrm{~mm}$ thick IN738 superalloy substrate with a $0.1 \mathrm{~mm}$ thick CoNiCrAlY bondcoat, were sintered under a temperature gradient. During the test, a $\mathrm{CO}_{2}$ laser was used to heat the coating surface to the target temperature (i.e. $1400{ }^{\circ} \mathrm{C}$ ). The temperature at the coating surface was monitored continuously using a thermal couple. The sample was held on a metal stage that was air-cooled. The temperature at the $\mathrm{TBC} /$ bondcoat interface was inferred by linearly interpolating the temperatures measured within in the substrate by embedded thermocouples that are positioned at three different distances from the interface. Three samples from the same batch were sintered at a surface temperature of $1400{ }^{\circ} \mathrm{C}$ and an interface temperature of $800{ }^{\circ} \mathrm{C}$ for $1 \mathrm{~h}, 4 \mathrm{hrs}$ and 8hrs respectively.

\subsection{Modulus determination}

Considering that the TBCs have been sintered under a temperature gradient, it is expected that the modulus varies with depth in the coating. The modulus profiles were determined using four-point bending tests, which were interpreted according to the Japanese Industrial Standard (JIS) H 8454 [4]. 
Test coupons measuring $55 \mathrm{~mm}(\mathrm{~L})$ x $7 \mathrm{~mm}(\mathrm{~W}) \times 3.5 \mathrm{~mm}(\mathrm{H})$ were prepared. The substrates were thinned to give a beam height of $3.5 \mathrm{~mm}$. The coupons were loaded under four-point bending with an outer span of $45 \mathrm{~mm}$ and an inner span of $15 \mathrm{~mm}$. The coupons were mounted in such a way that the $\mathrm{TBC}$ was under compression. A strain gauge, $5 \mathrm{~mm}$ long, was fixed to the centre of the TBC surface to monitor the strain at the TBC surface. The sample was loaded under displacement control with a loading rate of $0.01 \mathrm{~mm} / \mathrm{s}$ until the reading from the strain gauge reached 0.001 . The gradient of the load-surface strain curve was recorded before the sample was unloaded and re-loaded to the same surface strain 5 times for a repeatability check. The TBC surface was polished away progressively at $200 \mu \mathrm{m}$ increments and the same measurements were conducted at each TBC thickness. The actual thickness of each TBC layer polished away was determined by comparing the total height of the beam before and after polishing. Assuming that the modulus of each removed layer of TBC ( $200 \mu \mathrm{m}$ in thickness $)$ is constant, the value of the modulus for that layer was determined recursively.

Vertical cracks develop near the coating surface during sintering. In order to avoid the influence of these vertical cracks on the calibration of the sintering model, we have used the modulus values obtained at the middle of the coating, where the temperature during the holding period of the sintering test has been $1120 \pm 20^{\circ} \mathrm{C}$ assuming that the temperature has varied linearly from the surface to the interface during the sintering test.

\subsection{Model calibration against experimental measurement}

Sintering simulations have been run using the FE meshes shown in Figure 4. In order to reproduce the actual sintering condition, we have constrained the displacement along the horizontal (in-plane) direction at the vertical boundaries. The other boundary conditions are the same as described in Section 2.7. The same geometrical parameters as listed in Table 1 are used. 
The effective modulus determined using the image-based models prior to sintering is lower than the measured values for the as-deposited samples. Therefore, we have run 'pre-sintering' simulations and tracked the modulus evolution. We stopped the simulations when the effective modulus reached the values measured from the as-deposited TBCs. Sintering simulations were run using the *RESTART option in ABAQUS using results from the pre-sintering simulation as initial conditions.

The evolution of modulus normalised against that of the as-deposited TBC is compared in Figure S-2 for the model predictions and experimental measurements. A good fit to the experimental results was obtained by taking the effective boundary diffusivity $\Phi_{g}$ at $1300{ }^{\circ} \mathrm{C}$ as $6.3 \times 10^{-16} \mathrm{~mm}^{6} \mathrm{~mJ}^{-1} \mathrm{~s}^{-1}$ while keeping the other parameters unchanged. Segregation of impurities to grain boundaries can potentially increase the boundary diffusivity [5]. In fact, this updated value for the effective boundary diffusivity agrees with experimental data as reported in $[6,7]$.

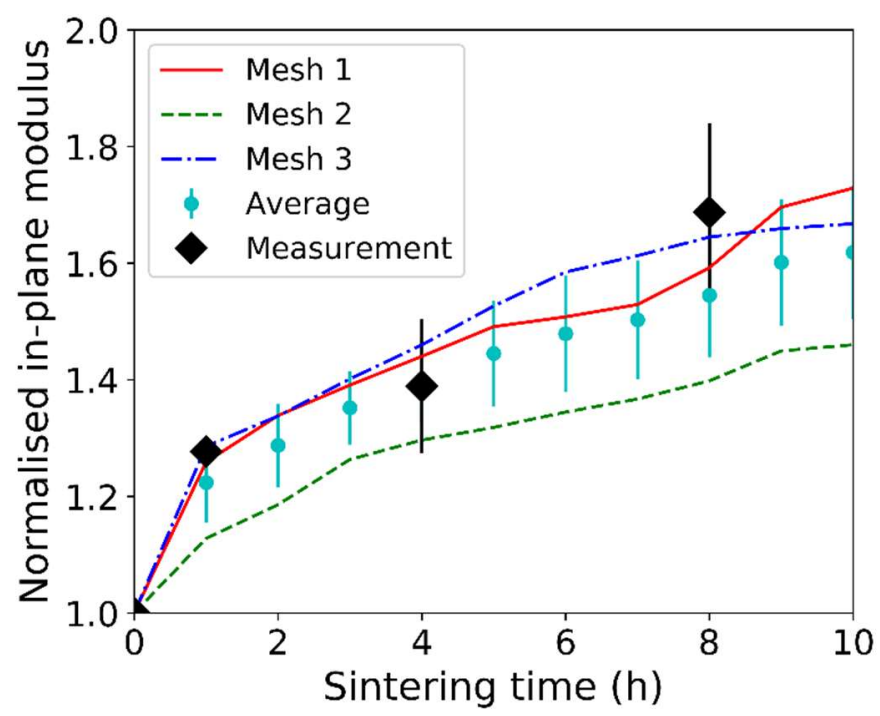

Figure S-2 Evolution of the measured elastic modulus normalised against that of the as-deposited TBC as a function of sintering time at $1120{ }^{\circ} \mathrm{C}$ : model results for three representative areas shown in Figure 4 and experimental measurements from the same batch of samples from which the SEM images in Figure 4 have been taken. The vertical error bars represent the standard deviation of results from the three 
meshes for the model results and the standard deviation of measurements respectively.

\section{Quantifying the cracks from micrographs}

The microstructure of the as-deposited and as-sintered $\left(1400^{\circ} \mathrm{C}, 8 \mathrm{hrs}\right.$ in air) $\mathrm{TBCs}$ were characterised by scanning electron microscope (SEM). For cross-sectional observations, the samples were first mounted in epoxy. The as-mounted samples were incrementally ground using $\mathrm{SiC}$ grinding paper with grit sizes of 400, 800 and 1200. Polishing was conducted using a diamond polishing paste with particle sizes of $6 \mu \mathrm{m}, 1 \mu \mathrm{m}$ and $0.25 \mu \mathrm{m}$ progressively. The polished samples were cleaned in an ultrasonic bath for $5 \mathrm{~min}$ and rinsed with ethanol. The asprepared specimens for SEM were coated with carbon ( 20nm). An FEI Quanta 650 FEG SEM was used to examine the microstructural features.

(a)

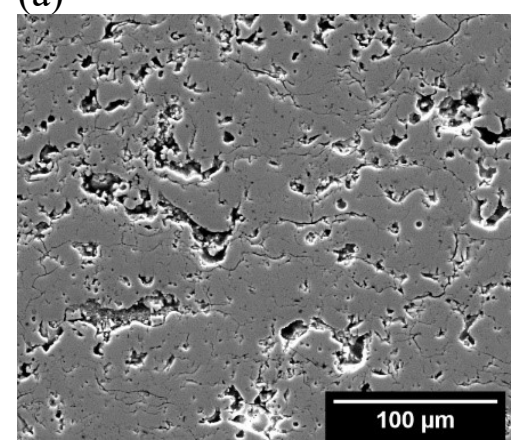

(d)

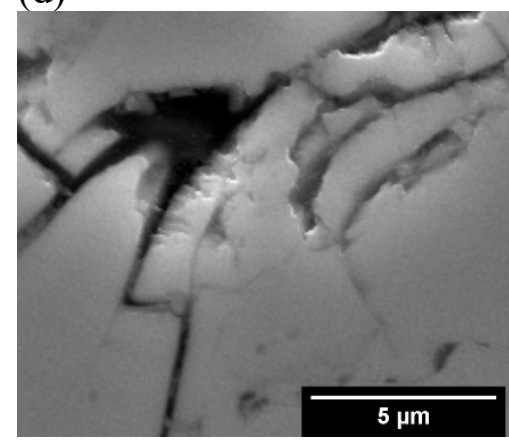

(b)

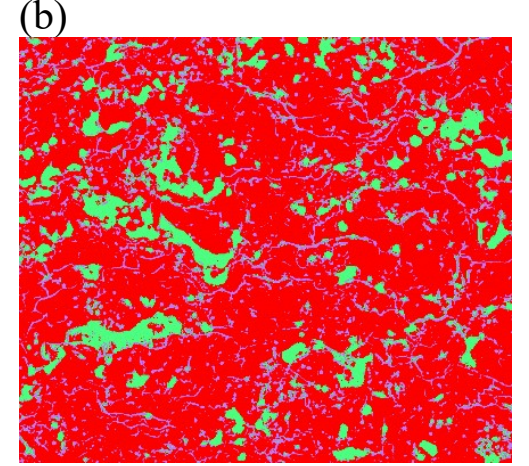

(e)

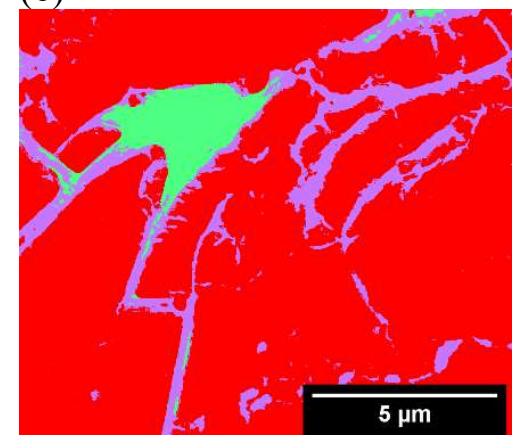

(c)

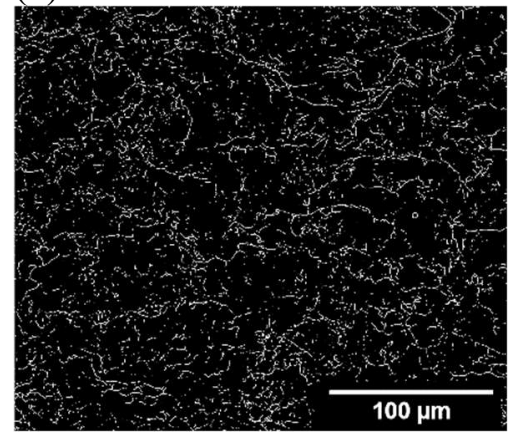

(f)

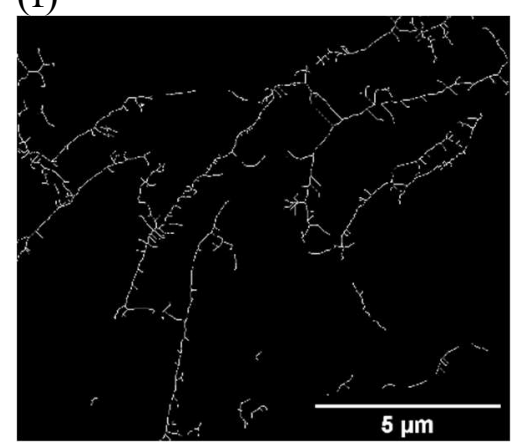

Figure S-3 SEM (SE mode) micrograph showing the polished cross-section of the as-deposited coating: (a) one RoI for crack orientation analysis and (d) zoom-in view on the typical inter-splat cracks and one pore trapped between the splats; (b) and (e) phase maps obtained from the trainable Weka segmentation plugin in ImageJ. Red is the YSZ matrix, Green is for the pores and Purple is for the cracks; (c) and (f) Central lines for the cracks determined by skeletonization. 
Pixels on the cross-sectional micrographs representing the cracks were identified using the trainable Weka segmentation plugin in the software ImageJ [8]. The central lines for the cracks were extracted using the Skeletonization plugin in ImageJ [9]. One example is shown in Figure S-3 (c). From the skeleton map, the vertex locations and length of each branch of the crack central lines was determined. The orientation distribution map can then be constructed. For each state, a region of interest (RoI) measuring 240 by $260 \mu \mathrm{m}^{2}$ (i.e. the size of the micrograph in the upper row in Figure S-2) has been analysed. Within the RoI, about 3800 crack segments were identified for the as-deposited sample and $\sim 3300$ for the as-sintered sample. In order to reduce the influence from noise, crack segments shorter than $1 \mu \mathrm{m}$ were removed.

\section{The geometric factor}

The additional strain rate due to the aligned cracks shown in Figure A-2 can be calculated as [10]:

$$
\begin{aligned}
& \dot{\varepsilon}_{33}^{c 1}=\sum_{\alpha=1}^{n} N_{\alpha} a_{\alpha}^{2} \dot{\varepsilon}_{33}^{\alpha}=f \dot{\varepsilon}_{33}^{\alpha}=-f \frac{16 c^{2}}{\pi a^{2}} P_{3} \Pi_{1111} \ln \left[\cos \left(\frac{\pi a}{2 c}\right)\right] \sigma_{33}^{\infty} \\
& \dot{\varepsilon}_{13}^{c 1}=\sum_{\alpha=1}^{n} N_{\alpha} a_{\alpha}^{2} \dot{\varepsilon}_{13}^{\alpha}=f \dot{\varepsilon}_{13}^{\alpha}=-f \frac{8 c^{2}}{\pi a^{2}} P_{1} \Pi_{1111} \ln \left[\cos \left(\frac{\pi a}{2 c}\right)\right] \sigma_{13}^{\infty}
\end{aligned}
$$

where

$$
\begin{aligned}
& P_{1}=\left\{\frac{1}{2 \Pi_{1111}}\left[2 \Pi_{1313}+\Pi_{1133}+\left(\Pi_{1111} \Pi_{3333}\right)^{\frac{1}{2}}\right]\right\}^{\frac{1}{2}} \\
& P_{3}=\frac{1}{2 \Pi_{1111}}\left\{2 \Pi_{3333}\left[2 \Pi_{1313}+\Pi_{1133}+\left(\Pi_{1111} \Pi_{3333}\right)^{\frac{1}{2}}\right]\right\}^{\frac{1}{2}}
\end{aligned}
$$

and $f \equiv N\left\langle a^{3}\right\rangle$ is the crack density parameter. The expressions for $\prod_{i j k l}$ are given in Eqn (S8). As a result, for $\chi \ll 1$, the overall creep compliance tensor for the cracked matrix reads: 


$$
\begin{aligned}
& \Pi_{3333}^{\prime}=\left\{1-\frac{1+\chi^{2}}{2 \chi^{2}} P_{3} f \frac{16 c^{2}}{\pi a^{2}} \ln \left[\cos \left(\frac{\pi a}{2 c}\right)\right]\right\} \frac{3 \Phi_{g}}{\chi h^{3}} \\
& \Pi_{1313}^{\prime}=\Pi_{2323}^{\prime}=\Pi_{1313}-P_{1} f \frac{8 c^{2}}{\pi a^{2}} \ln \left[\cos \left(\frac{\pi a}{2 c}\right)\right] \frac{3\left(1+\chi^{2}\right) \Phi_{g}}{2 \chi^{3} h^{3}}
\end{aligned}
$$

while the other components remain the same as in Eqn (S8).

The average opening rate for one crack under a traction $T_{n}$ normal to the surface over a length $a-l$ at the tips can be calculated as:

$$
\begin{aligned}
\left\langle\dot{w}_{n}\right\rangle & =-\frac{8}{\sqrt{2}} \frac{T_{n}}{a} g\left(\frac{a}{l}\right) P_{3}^{\prime} \Pi_{1111} \int_{0}^{a} \cos \left(\frac{\pi x_{1}}{2 c}\right) \int_{x_{1}}^{a} \tan \left(\frac{\pi \xi}{2 c}\right)\left\{\cos \left(\frac{\pi x_{1}}{c}\right)-\cos \left(\frac{\pi \xi}{c}\right)\right\}^{-1} d \xi d x_{1} \\
& =-T_{n} \frac{3 \Phi_{g} \pi a}{\chi h^{3}} G
\end{aligned}
$$

Here,

$$
P_{3}^{\prime}=\frac{1}{2 \Pi_{1111}^{\prime}}\left\{2 \Pi_{3333}^{\prime}\left[2 \Pi_{1313}^{\prime}+\Pi_{1133}^{\prime}+\left(\Pi_{111}^{\prime} \Pi_{3333}^{\prime}\right)^{\frac{1}{2}}\right]\right\}^{\frac{1}{2}}
$$

and

$$
G=\frac{4 \sqrt{2}}{\pi a^{2}} g\left(\frac{a}{l}\right) P_{3}^{\prime} \frac{\Pi_{1111}}{\prod_{3333}} \int_{0}^{a} \cos \left(\frac{\pi x_{1}}{2 c}\right) \int_{x_{1}}^{a} \tan \left(\frac{\pi \xi}{2 c}\right)\left\{\cos \left(\frac{\pi x_{1}}{c}\right)-\cos \left(\frac{\pi \xi}{c}\right)\right\}^{-1} d \xi d x_{1}
$$




\section{References}

[1] A.C.F. Cocks, S.P.A. Gill, J.Z. Pan, Modeling microstructure evolution in engineering materials, Adv Appl Mech 36 (1999) 81-162.

[2] G.W. Greenwood, A Formulation for Anisotropy in Diffusional Creep, P Roy Soc Lond a Mat 436(1896) (1992) 187-196.

[3] A. Cocks, N. Fleck, S. Lampenscherf, A brick model for asperity sintering and creep of APS TBCs, J Mech Phys Solids 63 (2014) 412-431.

[4] JIS H8454, Testing method for longitudinal elastic modulus of thermal barrier coatings, JSA, 2010.

[5] A. Cipitria, I.O. Golosnoy, T.W. Clyne, A sintering model for plasma-sprayed zirconia TBCs. Part I: Free-standing coatings, Acta Mater 57(4) (2009) 980-992.

[6] M.Z. Berbon, T.G. Langdon, An examination of the flow process in superplastic yttria-stabilized tetragonal zirconia, Acta Mater 47(8) (1999) 2485-2495.

[7] N. Balasubramanian, T.G. Langdon, Flow processes in superplastic yttria-stabilized zirconia: A Deformation Limit Diagram, Mat Sci Eng a-Struct 409(1-2) (2005) 46-51.

[8] I. Arganda-Carreras, V. Kaynig, C. Rueden, K.W. Eliceiri, J. Schindelin, A. Cardona, H.S. Seung, Trainable Weka Segmentation: a machine learning tool for microscopy pixel classification, Bioinformatics 33(15) (2017) 2424-2426.

[9] I. Arganda-Carreras, R. Fernandez-Gonzalez, A. Munoz-Barrutia, C. Ortiz-De-Solorzano, 3D Reconstruction of Histological Sections: Application to Mammary Gland Tissue, Microsc Res Techniq 73(11) (2010) 1019-1029.

[10] Section 6 - Elastic Solids with Microcracks, in: S. Nemat-Nasser, M. Hori (Eds.), North-Holland Series in Applied Mathematics and Mechanics, North-Holland1993, pp. 113-190. 